\title{
A Simple and Efficient Procedure for Knoevenagel Reaction Promoted by Imidazolium-based Ionic Liquids
}

\author{
Xiaomei $\mathrm{Hu}^{1}$, Conelius Ngwa \& Qinguo Zheng* \\ School of Life and Health Sciences, Aston University, Aston Street, \\ Birmingham, B4 7ET, United Kingdom
}

*Corresponding author: School of Life and Health Sciences, Aston University, Aston Street, Birmingham, B4 7ET, United Kingdom.

Email: q.zheng@aston.ac.uk; Telephone number: +44 (0)121 2044048. 


\section{Abstract}

Various room temperature ionic liquids (RTILs), notably,

1-methoxyethyl-3-methylimidazolium trifluoroacetate $[\mathrm{MeOEtMIM}]^{+}\left[\mathrm{CF}_{3} \mathrm{COO}\right]^{-}$, have been used to promote the Knoevenagel condensation to afford substituted olefins. All reactions proceeded effectively in the absence of any other catalysts or co-solvents with good to excellent yields. This method is simple and applicable to reactions involving a wide range of aldehydes and ketones with methylene compounds. The ionic liquid can be recycled without noticeable reduction of its catalytic activity. A plausible reaction mechanism is proposed.

\section{Graphical Abstract}

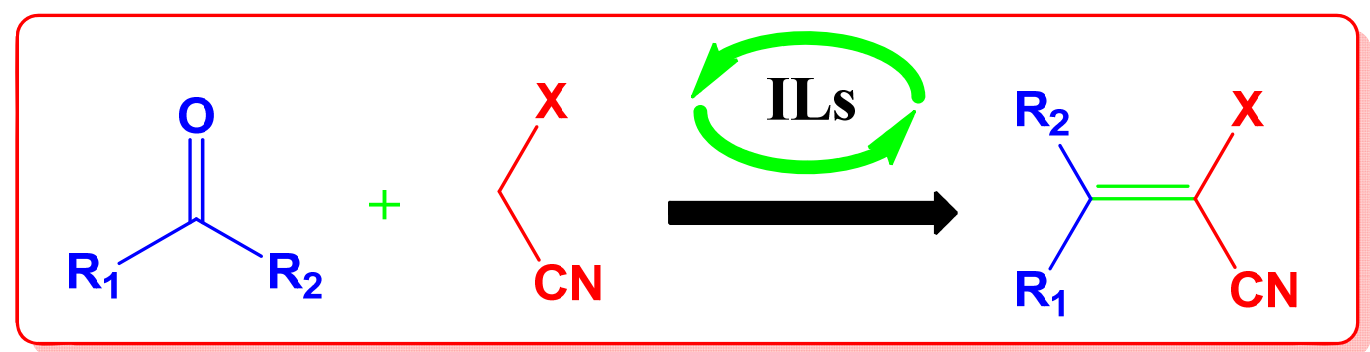

Imidazolium-based Ionic Liquids have been used to promote the Knoevenagel condensation to afford substituted olefins in good to excellent yields

Keywords: carbonyl compounds; catalysis; ionic liquids; Knoevenagel condensation; olefins. 


\section{Introduction}

The Knoevenagel condensation reaction is a widely employed method for carbon-carbon bond formation in organic reactions [1]. Traditionally the reaction is performed in organic solvents under homogenous conditions and in the presence of weak bases, such as ethylenediamine, pyridine, piperidine or an amino acid such as glycine, $\beta$-alanine and L-proline [1]. Recently a wide range of heterogeneous catalysts has also been used for the reaction such as natural phosphate [2], metal oxides [3], modified silica [4], calcined hydrotalcites [5], alkali metals-exchanged zeolites [6], aluminophosphates oxynitrides [7], alkaline earth carbonates [8] and sulphated $\mathrm{ZrO} 2$ [9]. In most cases, a combination of a catalyst and an organic solvent is used.

Recently, room temperature ionic liquids (RTILs) have gained wide popularity for their increasing applications in the areas of synthetic and biological chemistry. This is because they possess a number of interesting properties, especially their lack of vapor pressure, low flammability, a widely accessible temperature range and ease of reuse [10-14]. They are therefore considered to be environmentally friendly reaction media, which has generated an increasing interest in the application of RTILs for the Knoevenagel condensation reactions. For example, Salunkhe et al. have reported the Knoevenagel reactions in Lewis acidic ionic liquids 1-butyl-3-methylimidazolium chloroaluminate and 1-butylpyridinium chloroaluminate [15]. Base-catalysed Knoevenagel condensation in various ionic liquids has been documented, such as with proline [16, 17] and glycine [18-20]. In addition, Khan et al. have described the Knoevenagel reaction in ionic liquids catalysed by hydrotalcite [21]. Shen and co-workers have prepared a functionalized imidazolium cation-based ionic liquid immobilized on to silica gel, resulting in a solvent free system for the Knoevenagel reaction [22]. A guanidium ionic liquid [23] and 2-pyrrolidinecarboxylic acid ionic liquid [24] have been synthesized and used as catalysts for the Knoevenagel reaction. The Knoevenagel reaction has also been performed in water catalysed by ionic liquids based on 1,4-diazobicyclo[2.2.2]octane [25, 26]. Furthermore, use of dual functional ionic liquids for the condensation has been communicated by various research groups $[27,28]$. We have also previously reported the Knoevenagel reaction in various ionic liquids [29-31].

However it is worthwhile to note that the aforementioned approaches have various drawbacks, including the necessity to carry out procedures in an inert atmosphere [32], harsh reaction conditions [22], long reaction times and low yields of products [17, 19]. Some approaches 
need to use catalysts in addition to ionic liquids [17-20,31]. Moreover, in the most reported approaches, no ketones are involved since sterically hindered ketones are less reactive reagents than aldehydes in the Knoevenagel reaction [17-24, 26-30, 32-37].

In our continued efforts to use ionic liquids as environmentally friendly reaction media [29-31, 38], we have accomplished the use of various room temperature ionic liquids (RTILs), notably, 1-methoxyethyl-3-methylimidazolium trifluoroacetate $[\mathrm{MeOEtMIM}]^{+}\left[\mathrm{CF}_{3} \mathrm{COO}^{-}\right.$, as efficient recyclable catalysts and reaction mediums for the Knoevenagel condensation. The reactions not only involve aldehydes but also ketones to afford substituted olefins in good to excellent yields under mild reaction conditions. The ionic liquid $[\mathrm{MeOEtMIM}]^{+}\left[\mathrm{CF}_{3} \mathrm{COO}\right]^{-}$ can be recycled for up to five times with a minimal reduction in its catalytic activity. A plausible reaction mechanism is also proposed.

\section{Results and Discussion}

Ionic liquids 3a-h (Table 1) were synthesised from imidazole derivatives 1a-1c through commonly used quaternization reaction [39, 40] followed by anion metathesis [41]. The imidazole derivatives 1a-c were reacted with appropriate halides to afford halide salts 2a-e under anhydrous conditions. Treatment of these halide salts with an appropriate metal salt or an acid in various solvents afforded the required ionic liquids $\mathbf{3 a - h}$ in good to excellent yields. The specific methods for the synthesis of these ionic liquids are described in the Experimental Section. 
Table 1. Synthesis of ionic liquids

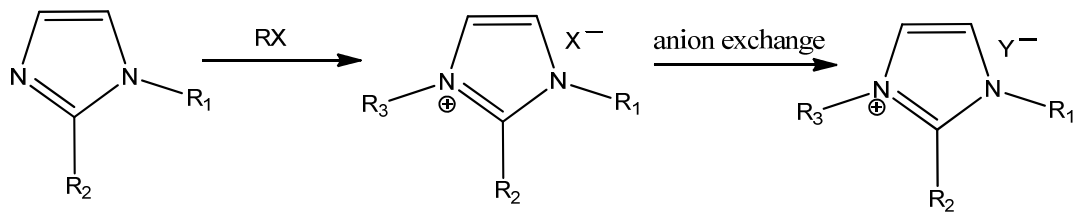

1a-c

2a-f

3a-i

\begin{tabular}{|c|c|c|c|c|c|}
\hline Compound No & $\mathbf{R}_{1}$ & $\mathbf{R}_{2}$ & $\mathbf{R}_{3}$ & $\mathbf{X}^{-}$ & $\mathbf{Y}^{-}$ \\
\hline $1 \mathbf{a}$ & $\mathrm{CH}_{3}$ & $\mathrm{H}$ & & & \\
\hline $1 b$ & $\mathrm{CH}_{3}$ & $\mathrm{CH}_{3}$ & & & \\
\hline 1c & $\mathrm{n}-\mathrm{Bu}$ & $\mathrm{H}$ & & & \\
\hline $2 \mathbf{a}$ & $\mathrm{CH}_{3}$ & $\mathrm{H}$ & $\mathrm{n}-\mathrm{Bu}$ & $\mathrm{Cl}$ & \\
\hline $2 \mathbf{b}$ & $\mathrm{CH}_{3}$ & $\mathrm{CH}_{3}$ & $\mathrm{n}-\mathrm{Bu}$ & $\mathrm{Cl}$ & \\
\hline $2 c$ & $\mathrm{CH}_{3}$ & $\mathrm{H}$ & $\mathrm{MeOMeOEt}$ & $\mathrm{Br}$ & \\
\hline $2 d$ & $\mathrm{CH}_{3}$ & $\mathrm{H}$ & $\mathrm{MeOEt}$ & $\mathrm{Cl}$ & \\
\hline $2 \mathbf{e}$ & $\mathrm{n}-\mathrm{Bu}$ & $\mathrm{H}$ & $\mathrm{MeOEt}$ & $\mathrm{Cl}$ & \\
\hline Зa & $\mathrm{CH}_{3}$ & $\mathrm{H}$ & $\mathrm{n}-\mathrm{Bu}$ & & $\mathrm{BF}_{4}$ \\
\hline $3 \mathbf{b}$ & $\mathrm{CH}_{3}$ & $\mathrm{H}$ & $\mathrm{n}-\mathrm{Bu}$ & & $\mathrm{PF}_{6}$ \\
\hline $3 c$ & $\mathrm{CH}_{3}$ & $\mathrm{H}$ & $\mathrm{n}-\mathrm{Bu}$ & & $\mathrm{CF}_{3} \mathrm{COO}$ \\
\hline 3d & $\mathrm{CH}_{3}$ & $\mathrm{CH}_{3}$ & $\mathrm{n}-\mathrm{Bu}$ & & $\mathrm{CF}_{3} \mathrm{COO}$ \\
\hline $3 \mathbf{e}$ & $\mathrm{CH}_{3}$ & $\mathrm{H}$ & $\mathrm{MeOMeOEt}$ & & $\mathrm{CF}_{3} \mathrm{COO}$ \\
\hline $3 f$ & $\mathrm{CH}_{3}$ & $\mathrm{H}$ & $\mathrm{MeOEt}$ & & $\mathrm{CF}_{3} \mathrm{COO}$ \\
\hline $3 g$ & $\mathrm{CH}_{3}$ & $\mathrm{H}$ & $\mathrm{MeOEt}$ & & $\mathrm{CF}_{3} \mathrm{SO}_{3}$ \\
\hline $3 \mathbf{h}$ & $\mathrm{n}-\mathrm{Bu}$ & $\mathrm{H}$ & $\mathrm{MeOEt}$ & & $\mathrm{CF}_{3} \mathrm{SO}_{3}$ \\
\hline \multicolumn{6}{|c|}{ 3a: 1-Butyl-3-methylimidazolium tetrafluoroborate $\left\{[\mathrm{BMIM}]^{+}\left[\mathrm{BF}_{4}\right]^{-}\right\}$} \\
\hline \multicolumn{6}{|c|}{ 3b: 1-Butyl-3-methylimidazolium hexafluorophosphate $\left\{[\mathrm{BMIM}]^{+}\left[\mathrm{PF}_{6}\right]^{-}\right\}$} \\
\hline \multicolumn{6}{|c|}{ 3c: 1-Butyl-3-methylimidazolium trifluoroacetate $\left\{[\mathrm{BMIM}]^{+}\left[\mathrm{CF}_{3} \mathrm{COO}^{-}\right\}\right.$} \\
\hline \multicolumn{6}{|c|}{ 3d:1-Butyl-2,3-dimethylimidazolium trifluoroacetate $\left.\left\{[\mathrm{BMMIM}]^{+} \mathrm{CF} 3 \mathrm{COO}\right]^{-}\right\}$} \\
\hline \multicolumn{6}{|c|}{ 3e:1-Methoxymethoxyethyl-3-methylimidazolium trifluoroacetate $\left\{[\mathrm{MeOMeOEtMIM}]^{+}\left[\mathrm{CF}_{3} \mathrm{COO}^{-}\right\}\right.$} \\
\hline \multicolumn{6}{|c|}{ 3f: 1-Methoxyethyl-3-methylimidazolium trifluoroacetate $\left\{[\mathrm{MeOEtMIM}]^{+}\left[\mathrm{CF}_{3} \mathrm{COO}^{-}\right\}\right.$} \\
\hline \multicolumn{6}{|c|}{ 3g: 1-Methoxyethyl-3-methylimidazolium trifluoromethanesulfonate $\left\{[\mathrm{MeOEtMIM}]^{+}\left[\mathrm{CF}_{3} \mathrm{SO}_{3}\right]^{-}\right\}$} \\
\hline \multicolumn{6}{|c|}{ 3h: 1-Methoxyethyl-3-butylimidazolium trifluoromethanesulfonate $\left\{[\mathrm{MeOEtBuIM}]^{+}\left[\mathrm{CF}_{3} \mathrm{SO}_{3}\right]^{-}\right\}$} \\
\hline
\end{tabular}

A number of solvents have been reported for the quaternization reactions [42, 43]. In general, it will help to drive the reaction to completion and help the product isolation if a resulting ionic liquid is immiscible with the solvent. In order to identify suitable solvents for the quaternization reaction various solvents were investigated for the reaction between 1-methylimidazole and 1-chlorobutane. The results are shown in Figure 1. 


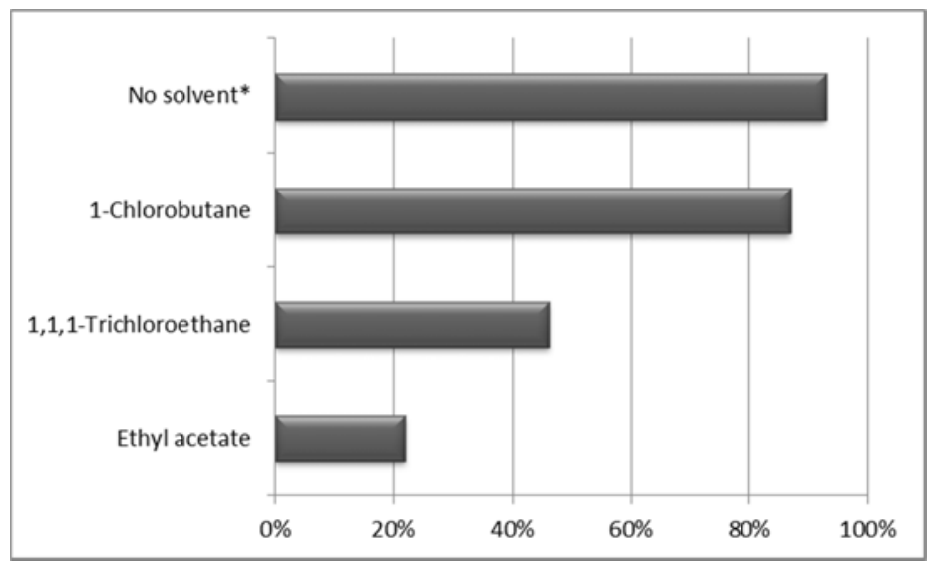

Figure 1. Comparison of various solvents for the preparation of $[\mathrm{BMIM}]^{+} \mathrm{Cl}^{-}(2 \mathrm{a})\left({ }^{*}\right.$ equal molar of the starting materials without addition of other solvents).

The above data show that when chlorobutane was used as a solvent the yield was higher than that in other solvents. Additionally, the quaternization reaction with no solvent also proceeded well with an excellent yield. Consequently all quaternization reactions were performed in an appropriate halide reagent without the addition of other solvents. These halides salts (2a-e) were characterized with ${ }^{1} \mathrm{H} \mathrm{NMR},{ }^{13} \mathrm{C} \mathrm{NMR}$, IR and MS before being used for the preparation of ionic liquids (3a-h) through anion exchange reactions (Table 1).

It is highly desirable to remove any trace of 1-alkylimidazoles from the quaternization products as they could play an unfavorable role in some applications of ionic liquids. For example, electrophilic catalysts can coordinate to 1-alkylimidazoles in an irreversible manner, resulting in deactivation of the catalysts [44]. One commonly used method to remove alkylimidazoles is by heating in vacuo in a rotary evaporator. But this method is not always efficient as revealed by NMR analysis of $[\mathrm{BMIM}]^{+} \mathrm{Cl}^{-}$obtained after drying at $80{ }^{\circ} \mathrm{C}$ for $8 \mathrm{~h}$ in vacuo (Fig. 2a), showing the presence of residue 1-methylimidazole. Therefore, an alternative approach was used. This was to dissolve the halide salt in dry acetonitrile at $50{ }^{\circ} \mathrm{C}$ and add the solution to a mixture of dry acetonitrile/diethyl ether cooled in an ice bath under stirring. ${ }^{1} \mathrm{H}$ NMR spectrum (Fig. 2b) of $[\mathrm{BMIM}]^{+} \mathrm{Cl}^{-}$obtained using this method confirmed that there was no residual 1-methylimidazole. Subsequently, all solid halide salts were purified by recrystallization before anion exchange reactions. 


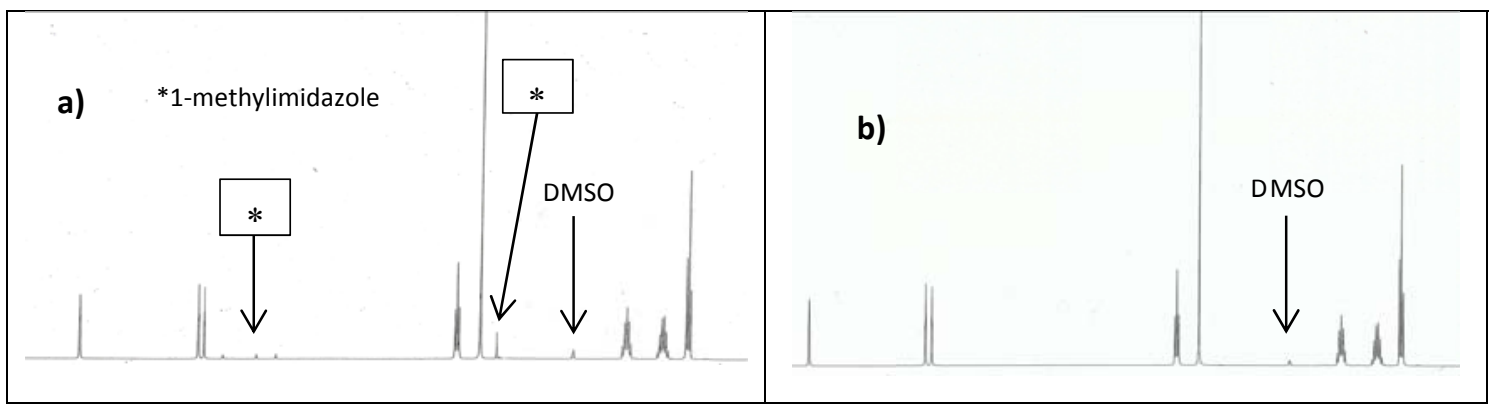

Figure 2. ${ }^{1}$ H NMR spectrum of 1-butyl-3-methylimidazolium chloride, a) purified by heating under high vacuum; b) by recrystallization.

$[\mathrm{BMIM}]^{+}\left[\mathrm{BF}_{4}\right]^{-}$(3a) was prepared as described [45] with some modifications. $[\mathrm{BMIM}]^{+} \mathrm{Cl}^{-}$ (2a) was dissolved in water and tetrafluoroboric acid (1 equivalent) was added slowly under stirring. The mixture was then stirred for 24 hours at room temperature. The resulting ionic liquid was extracted with chilled dichloromethane. The extract was washed with chilled water until the aqueous phase was $\mathrm{pH}$ neutral and free of chloride ion $\left(\mathrm{AgNO}_{3}\right)$. It was not efficient to extract water miscible ionic liquids $[\mathrm{BMIM}]^{+}\left[\mathrm{BF}_{4}\right]^{-}$from the aqueous solution so large volume of extraction solvent was used initially. However, it was noticed later the extraction efficiency improved significantly when carried out at a low temperature. Consequently, the reaction mixture and $\mathrm{CH}_{2} \mathrm{Cl}_{2}$ were chilled in an ice bath before the extraction. [BMIM] $]^{+}\left[\mathrm{PF}_{6}\right]^{-}$ (3b) is a water-immiscible ionic liquid. Its preparation was carried out in aqueous solution by the reaction between $\mathrm{KPF}_{6}$ with $[\mathrm{BMIM}]^{+}[\mathrm{Cl}]^{-}(\mathbf{2 a})$ at room temperature [46]. A biphasic layer was formed when the reaction was complete. The aqueous layer was separated off and the organic layer was washed with water to give a pure product after being dried at $60{ }^{\circ} \mathrm{C}$ in vacuo.

Ionic liquids 3c-i were prepared through anion metathesis reactions of an appropriate halide salt with either $\mathrm{CF}_{3} \mathrm{COONa}$ or $\mathrm{CF}_{3} \mathrm{SO}_{3} \mathrm{Na}$ in acetonitrile or acetone [47]. Both $\mathrm{CF}_{3} \mathrm{COONa}$ and $\mathrm{CF}_{3} \mathrm{SO}_{3} \mathrm{Na}$ can be dissolved in acetonitrile or acetone. The reactions were completed with the resulting sodium halides precipitating out spontaneously. An initial attempt was made to synthesise these ionic liquids in an aqueous medium but with unsatisfactory yields, mainly due to poor extraction efficiency of these water soluble ionic liquids with organic solvents in aqueous solution. These ionic liquids were characterized with ${ }^{1} \mathrm{H} \mathrm{NMR},{ }^{13} \mathrm{C}$ NMR and MS.

It has been widely reported that the C-2 hydrogen of imidazolium based ionic liquids plays a significant role in various organic reactions promoted by ionic liquids [48-51]. In order to 
investigate its involvement in promoting the Knoevenagel reaction, ionic liquid 3d was prepared, in which the C-2 hydrogen was substituted with a methyl group.

The ionic liquids 3a-h prepared above were examined and compared to evaluate their efficiency for promoting the Knoevenagel reaction as outlined in Scheme 1. Benzaldehyde 4 and ethyl cyanoacetate 5 were mixed and one of the ionic liquids added and the mixture stirred for an appropriate time at $25^{\circ} \mathrm{C}$ (Table 2). The product 6 was extracted with diethyl ether. After removal of the solvent the crude product was purified by recrystallization. The results summarised in Table 2 demonstrate that Knoevenagel condensation of benzaldehyde with ethyl cyanoacetate proceeded in all of the RTILs tested but with significantly variable efficiency. By comparing the results, it is evident that the structures of both the imidazolium moiety and its counter anion in the ionic liquids have a significant effect on their activity for promoting the Knoevenagel reaction. Particularly, a substantial reduction in the catalytic efficiency was observed when the C-2 hydrogen was replaced by a methyl group (entries 4).

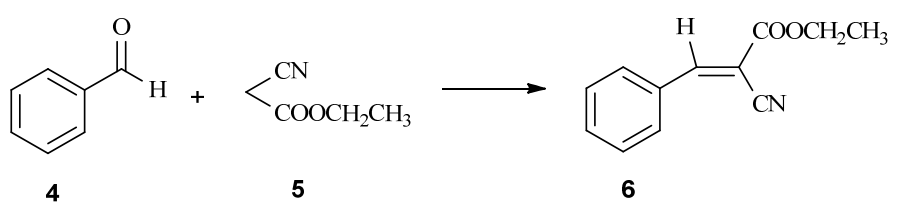

Scheme 1. Reaction of benzaldehyde with ethyl cyanoacetate in various ILs.

Table 2. Knoevenagel condensation of benzaldehyde with ethyl cyanoacetate in various ionic liquids ${ }^{\mathrm{a}}$

\begin{tabular}{|c|c|c|c|}
\hline Entry & Solvent & Reaction time & Yield (\%) ${ }^{b}$ \\
\hline 1 & {$[\mathrm{BMIM}]^{+}\left[\mathrm{BF}_{4}\right]^{-}(\mathbf{3 a})$} & $24 \mathrm{~h}$ & 85 \\
\hline 2 & {$\left[\mathrm{BMIM}^{+}\left[\mathrm{PF}_{6}\right]^{-}(3 \mathbf{b})\right.$} & $22 \mathrm{~h}$ & 63 \\
\hline 3 & {$[\mathrm{BMIM}]^{+}\left[\mathrm{CF}_{3} \mathrm{COO}^{-}(3 \mathrm{c})\right.$} & $1 \mathrm{~h}$ & 98 \\
\hline 4 & {$\left[\mathrm{BMMIM}^{+}[\mathrm{CF} 3 \mathrm{COO}]^{-}\right.$(3d) } & $4 \mathrm{~h}$ & 62 \\
\hline 5 & {$[\mathrm{MeOMeOEtMIM}]^{+}\left[\mathrm{CF}_{3} \mathrm{COO}\right]^{-}(\mathbf{3 e})$} & $40 \mathrm{~min}$ & 95 \\
\hline 6 & {$\left[[\mathrm{MeOEtMIM}]^{+}\left[\mathrm{CF}_{3} \mathrm{COO}^{-}\right.\right.$(3f) } & $40 \mathrm{~min}$ & 98 \\
\hline 7 & {$[\mathrm{MeOEtMIM}]^{+}\left[\mathrm{CF}_{3} \mathrm{SO}_{3}\right]^{-}(\mathbf{3 g})$} & $1 \mathrm{~h}$ & 81 \\
\hline 8 & {$[\mathrm{MeOEtBuIM}]^{+}\left[\mathrm{CF}_{3} \mathrm{SO}_{3}\right]^{-}(\mathbf{3 h})$} & $2 \mathrm{~h}$ & 54 \\
\hline
\end{tabular}

It has been reported widely that the catalytic efficiency of ILs for various organic reactions is influenced by the structures of the cation and the counter anion. Chakraborti and co-workers reported that catalytic activity of ILs derived from 1-methyl-3-alkylimidazolium cations is affected by both cations and anoins for various organic reactions [48, 50, 51]. The 
involvement of the hydrogen bond donor of C-2 hydrogen in the imidazolium moiety and the hydrogen bond acceptor of the counter anion was supported by the results of various spectroscopic studies. The importance of the C-2 hydrogen of the imidazolium species was further demonstrated through a large reduction of catalytic efficiency of ILs derived from imidazolium cations having an alkyl group substituted at C-2 [48, 50, 51]. Zhu et al.[27] observed the importance of both the cations and the counter anions in N, N-dimethyl ethanolammonium-based ionic liquids. Their results demonstrated that the increase in the hydrogen bond donor ability of the cation and the hydrogen bond acceptor ability of the anion in an ionic liquid can lead to an enhancement in its catalytic activity for the Knoevenagel condensation. A possible catalytic mechanism was proposed, which involved the activation of $\mathrm{C}=\mathrm{O}$ group through the formation of a hydrogen bond with the cations of the ILs and an attack by the anions of the ILs to the active methylene compounds to form a carboanion [27].

Based on the different catalytic activities observed for the ILs investigated here, particularly the importance of the involvement of C-2 hydrogen in the imidazolium moiety (entry 4) and the observations and reaction mechanism reported by others $[27,48,50,51]$ a possible mechanism is proposed and illustrated in Scheme 2. It involves an attack to the active methylene compound by the counter anion of an IL to form a carboanion. This anion then attacks the $\mathrm{C}=\mathrm{O}$ group of benzaldehyde, which has been activated by the formation of a hydrogen bond with the C-2 hydrogen of the imidazolium cations, to produce an alcohol intermediate. An attack to this intermediate by the anion of the ILs produce the final dehydration product. In this reaction mechanism, the counter anions of ionic liquids play an activating role for the nucleophiles of methylene compounds to produce carboanions and the imidazolium cations act as activators for the electrophiles of carbonyl compounds by increasing the polarisation of $\mathrm{C}=\mathrm{O}$ bond through the formation of a hydrogen bond with the C-2 hydrogen. 


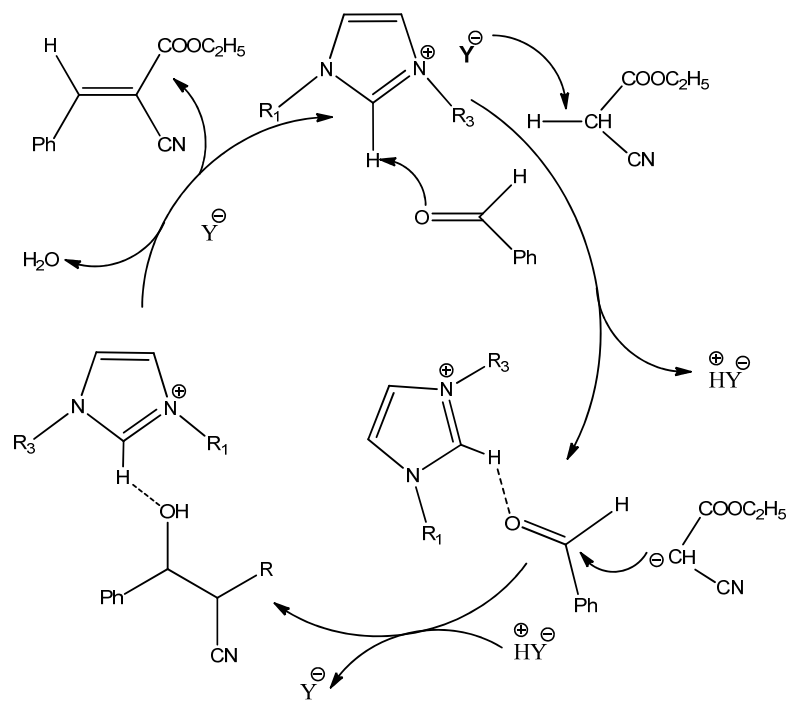

Scheme 2. Proposed mechanism for the Knoevenagel reaction.

Although all the ionic liquids tested demonstrated activity for promoting Knoevenagel reactions (Table 2), an ideal ionic liquid for promoting the Knoevenagel condensation reaction should have a strong catalytic activity, a low viscosity and be economically viable for a large scale production. According to the yields and times of the reactions (Table 2), $[\mathrm{BMIM}]^{+}\left[\mathrm{CF}_{3} \mathrm{COO}\right]^{-}(\mathbf{3 c}),[\mathrm{MeOMeOEtMIM}]^{+}\left[\mathrm{CF}_{3} \mathrm{COO}^{-}(\mathbf{3 e})\right.$ and $[\mathrm{MeOEtMIM}]^{+}\left[\mathrm{CF}_{3} \mathrm{COO}\right]^{-}$(3f) seemed to be better choices compared with the others. However, $[\mathrm{MeOMeOEtMIM}]^{+}\left[\mathrm{CF}_{3} \mathrm{COO}^{-}(3 \mathbf{e})\right.$ is relatively more viscous and the starting materials for its preparation are more expensive than for that of the other two ionic liquids. A further experiment was thus carried out in order to differentiate the other two ionic liquids. When the reaction of 4-methylbenzaldehyde with ethyl cyanoacetate was carried out in $[\mathrm{BMIM}]^{+}\left[\mathrm{CF}_{3} \mathrm{COO}\right]^{-}(3 \mathrm{c})$, the reaction time was 30 minutes with a yield of $74 \%$, whereas the same reaction in $[\mathrm{MeOEtMIM}]^{+}\left[\mathrm{CF}_{3} \mathrm{COO}\right]^{-}$(3f) was 20 minutes with a yield of $89 \%$.

Considering these results, $\mathbf{3 f}$ was chosen as a dual functional ionic liquid for the Knoevenagel reaction. In order to further assess its catalytic activity a wide range of reactions between aldehydes or ketones (7) with methylene compounds (8) were investigated in $[\mathrm{MeOEtMIM}]^{+}\left[\mathrm{CF}_{3} \mathrm{COO}\right]^{-}$. The results are summarised in Table 3. 
Table 3. Knoevenagel condensation of aldehydes and ketones with methylene compounds in $[\mathrm{MeOEtMIM}]^{+}\left[\mathrm{CF}_{3} \mathrm{COO}^{-\mathrm{a}}\right.$

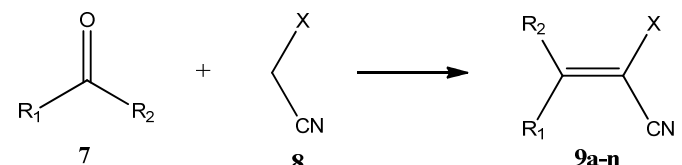

\begin{tabular}{|c|c|c|c|c|c|c|c|}
\hline Entry & R1 & $\mathbf{R} 2$ & $\mathbf{X}$ & Time & Temperature & Yield (\%) & Product \\
\hline 1 & $\mathrm{Ph}$ & $\mathrm{H}$ & COOEt & $40 \mathrm{~min}$ & $25^{\circ} \mathrm{C}$ & 98 & $9 a$ \\
\hline 2 & $4-\mathrm{Cl}-\mathrm{C}_{6} \mathrm{H}_{4}$ & $\mathrm{H}$ & COOEt & $5 \mathrm{~min}$ & $25^{\circ} \mathrm{C}$ & 97 & $9 b$ \\
\hline 3 & $4-\mathrm{Me}-\mathrm{C}_{6} \mathrm{H}_{4}$ & $\mathrm{H}$ & COOEt & $20 \mathrm{~min}$ & $25^{\circ} \mathrm{C}$ & 89 & $9 \mathrm{c}$ \\
\hline 4 & $4-\mathrm{NO}_{2}-\mathrm{C}_{6} \mathrm{H}_{4}$ & $\mathrm{H}$ & COOEt & $10 \mathrm{~min}$ & $25^{\circ} \mathrm{C}$ & 81 & $9 \mathrm{~d}$ \\
\hline 5 & 4-HOOC- $\mathrm{C}_{6} \mathrm{H}_{4}$ & $\mathrm{H}$ & COOEt & $50 \mathrm{~min}$ & $25^{\circ} \mathrm{C}$ & 77 & $9 \mathrm{e}$ \\
\hline 6 & 2-Naphthyl & $\mathrm{H}$ & COOEt & $15 \mathrm{~min}$ & $25^{\circ} \mathrm{C}$ & 98 & $9 \mathrm{f}$ \\
\hline 7 & $3-\mathrm{Cl}-\mathrm{C}_{6} \mathrm{H}_{4}$ & $\mathrm{H}$ & COOMe & $2 \min$ & $25^{\circ} \mathrm{C}$ & 99 & $9 g$ \\
\hline 8 & 4-Me- $\mathrm{C}_{6} \mathrm{H}_{4}$ & $\mathrm{H}$ & COOMe & $10 \mathrm{~min}$ & $25^{\circ} \mathrm{C}$ & 91 & $9 \mathrm{~h}$ \\
\hline 9 & $4-\mathrm{MeO}-\mathrm{C}_{6} \mathrm{H}_{4}$ & $\mathrm{H}$ & COOMe & $35 \mathrm{~min}$ & $25^{\circ} \mathrm{C}$ & 78 & $9 \mathrm{i}$ \\
\hline 10 & $4-\mathrm{HO}-\mathrm{C}_{6} \mathrm{H}_{4}$ & $\mathrm{H}$ & COOMe & $40 \mathrm{~min}$ & $25^{\circ} \mathrm{C}$ & 83 & $9 \mathrm{j}$ \\
\hline 11 & $-\left(\mathrm{CH}_{2}\right)_{4^{-}}$ & & COOMe & $48 \mathrm{~h}$ & $25^{\circ} \mathrm{C}$ & 85 & $9 \mathrm{k}$ \\
\hline 12 & $-\left(\mathrm{CH}_{2}\right)_{5^{-}}$ & & COOMe & $16 \mathrm{~h}$ & $25^{\circ} \mathrm{C}$ & 56 & 91 \\
\hline 13 & $\mathrm{Ph}$ & $\mathrm{CH}_{3}$ & COOMe & $48 \mathrm{~h}$ & $50^{\circ} \mathrm{C}$ & 55 & $9 \mathrm{~m}$ \\
\hline 14 & $\mathrm{Ph}$ & $\mathrm{CH}_{3}$ & COOEt & $48 \mathrm{~h}$ & $50^{\circ} \mathrm{C}$ & 47 & $9 n$ \\
\hline 15 & $\mathrm{n}-\mathrm{C}_{3} \mathrm{H}_{7}$ & $\mathrm{H}$ & COOEt & $2 \mathrm{~h}$ & $25^{\circ} \mathrm{C}$ & 82 & 90 \\
\hline 16 & 2-Furyl & $\mathrm{H}$ & COOEt & $80 \mathrm{~min}$ & $25^{\circ} \mathrm{C}$ & 85 & $9 p$ \\
\hline
\end{tabular}

As illustrated in Table 3, various aldehydes, including aromatic aldehydes (entries 1-10) with electron-donating groups (methyl, methoxyl and hydroxyl) or electron-withdrawing groups (chloro, nitro, carboxyl), aliphatic aldehyde (entry 15) and heterocyclic aldehyde (entry 16), reacted speedily with the active methylene compounds at $25{ }^{\circ} \mathrm{C}$ with satisfactory yields. In the case of ketones, longer reaction times were needed (entries 11-12). As ketones becomes more steric-hindrance both longer reaction times and higher temperature were applied to overcome the increased steric hindrance of the substrates (entries 13-14). All products were characterized by ${ }^{1} \mathrm{H}$ NMR and ${ }^{13} \mathrm{C}$ NMR. These reactions were usually stereoselective with the predominate products being the E-configuration, as reported previously in the literature $[31,52]$. However, two isomers (E \& Z) were obtained when acetophenone was reacted with methylene compounds but with E-geometry as the main product as revealed by ${ }^{1} \mathrm{H}$ NMR and ${ }^{13} \mathrm{C}$ NMR.

One of the main advantages of ionic liquids is their ease of reuse. In order to determine the 
performance of the recycled ionic liquid, the ionic liquid was recovered from the reaction mixture and reaction of benzaldehyde with ethyl cyanoacetate was carried out in the recycled $[\mathrm{MeOEtMIM}]^{+}\left[\mathrm{CF}_{3} \mathrm{COO}^{-}\right.$(3f). The results summarized in Table 4 demonstrate $\mathbf{3 f}$ was still effective after 5 cycles with a minimal reduction in its activity.

Table 4. Knoevenagel condensation of benzaldehyde with ethyl cyanoacetate

\begin{tabular}{|l|l|}
\hline Ionic liquid & Yield (\%) \\
\hline$[\mathrm{MeOEtMIM}]^{+}\left[\mathrm{CF}_{3} \mathrm{COO}\right]^{-}$(Fresh) & 98 \\
\hline$[\mathrm{MeOEtMIM}]^{+}\left[\mathrm{CF}_{3} \mathrm{COO}\right]^{-}$(cycle1) & 94 \\
\hline$[\mathrm{MeOEtMIM}]^{+}\left[\mathrm{CF}_{3} \mathrm{COO}\right]^{-}$(cycle2) & 91 \\
\hline$[\mathrm{MeOEtMIM}]^{+}\left[\mathrm{CF}_{3} \mathrm{COO}\right]^{-}$(cycle3) & 88 \\
\hline$[\mathrm{MeOEtMIM}]^{+}\left[\mathrm{CF}_{3} \mathrm{COO}\right]^{-}$(cycle4) & 85 \\
\hline$[\mathrm{MeOEtMIM}]^{+}\left[\mathrm{CF}_{3} \mathrm{COO}^{-}\right.$(cycle5) & 86 \\
\hline
\end{tabular}

\section{Conclusion}

In summary, we have developed a simple and efficient procedure for Knoevenagel condensation reactions in various ionic liquids. The results obtained with 1-methoxyethyl-3-methylimidazolium trifluoroacetate (3f) show that the approach is applicable to a large number of substrates, including not only aldehydes but also ketones, to afford substituted olefins in good to excellent yields in short reaction times. The approach also has the benefit of mild reaction temperatures and easy preparation procedures. In contrast to other reported procedures in which both ionic liquids and additional catalysts are needed [17-20, 31], the ionic liquids reported here act as both a catalyst and a solvent for the reactions and can be recycled and reused for 5 times without obvious loss of its catalytic activity. Furthermore, it has been demonstrated that the catalytic activity of the ionic liquids for the Knoevenagel reactions is likely linked to their ability to form hydrogen bonds. We believe this simple and green method will be a useful alternative to the existing Knoevenagel reaction procedures.

\section{Experimental section}

\subsection{General}

Chemicals and solvents were used as received without purification. Melting points were determined on a Reichert-Jung Galen micro melting point apparatus and were uncorrected. ${ }^{1} \mathrm{H}(250 \mathrm{MHz})$ and ${ }^{13} \mathrm{C}(62.9 \mathrm{MHz})$ NMR spectra were recorded on a Bruker AC-250 spectrometer with TMS as an internal standard. Infrared 
spectra were recorded on a Mattson 3000 FT-IR spectrophotometer using KBr discs for solids and thin films for liquids. Mass spectra were recorded on a Waters LCT Premier mass spectrometer by electron spray ionisation or electron impact ionisation. TLC was carried out on pre-coated Merck 60 F254 plates and visualized using UV (254 $\mathrm{nm}$ and $360 \mathrm{~nm})$.

\subsection{Synthesis of ionic liquids 3a-h}

\subsubsection{1-Butyl-3-methylimidazolium tetrafluoroborate $[\mathrm{BMIM}]^{+}\left[\mathrm{BF}_{4}\right]^{-}$(3a) $[45]$}

1-butyl-3-methylimidazolium chloride $\left[\mathrm{BMIM}^{+} \mathrm{Cl}^{-}\right.$(2a) [46]: 1-Methylimidazole (1a, $\left.80 \mathrm{~mL}, 1 \mathrm{~mol}\right)$ was added dropwise to 1 -chlorobutane $(200 \mathrm{~mL}, 1.9 \mathrm{~mol})$. The mixture was stirred vigorously and refluxed at $80{ }^{\circ} \mathrm{C}$ for 24 h. When the reaction was complete, the excess 1-chlorobutane was decanted and the crude ionic liquid was washed with chlorobutane $(2 \times 20 \mathrm{~mL})$. The trace of remaining 1-chlorobutane was removed with rotary evaporation at $60{ }^{\circ} \mathrm{C}$ for $30 \mathrm{~min}$, followed by at $80{ }^{\circ} \mathrm{C}$ for $4 \mathrm{~h}$ under reduced pressure. The crude product was further purified by recrystallization (acetonitrile/ether) and dried under vacuum to afford 2a as a white solid (151.9 g, 87\%). ${ }^{1} \mathrm{H}$ NMR (250 MHz, DMSO-d $\left.{ }_{6}\right) \delta: 9.57$ (s, 1H, NCHN), 7.91 (t, J=1.90 Hz, 1H, NCHCHN), $7.83(\mathrm{t}, J=1.90 \mathrm{~Hz}, 1 \mathrm{H}, \mathrm{NHCCHN}), 4.21\left(\mathrm{t}, J=6.95 \mathrm{~Hz}, 2 \mathrm{H}, \mathrm{NCH}_{2}\right), 3.88\left(\mathrm{~s}, 3 \mathrm{H}, \mathrm{N}-\mathrm{CH}_{3}\right), 1.76$ (quint, $J=7.58$ $\mathrm{Hz}, 2 \mathrm{H}, \mathrm{NCH}_{2} \mathrm{CH}_{2}$ ), 1.23 (sext, $J=7.58 \mathrm{~Hz}, 2 \mathrm{H}, \mathrm{NCH}_{2} \mathrm{CH}_{2} \mathrm{CH}_{2}$ ), 0.88 (t, $J=7.58 \mathrm{~Hz}, 3 \mathrm{H}, \mathrm{N}-\mathrm{CH}_{2} \mathrm{CH}_{2} \mathrm{CH}_{2}-\mathrm{CH}_{3}$ ).

${ }^{13} \mathrm{C}$ NMR (62.9 MHz, DMSO-d 6 ) $\delta: 136.66$ (NCHN), 123.47 (NCHCHN), 122.19 (NCHCHN), 48.27

$\left(\mathrm{NCH}_{2} \mathrm{CH}_{2} \mathrm{CH}_{2} \mathrm{CH}_{3}\right), 35.60\left(\mathrm{NCH}_{3}\right), 31.31\left(\mathrm{NCH}_{2} \mathrm{CH}_{2} \mathrm{CH}_{2} \mathrm{CH}_{3}\right), 18.67\left(\mathrm{NCH}_{2} \mathrm{CH}_{2} \mathrm{CH}_{2} \mathrm{CH}_{3}\right), 13.21$ $\left(\mathrm{NCH}_{2} \mathrm{CH}_{2} \mathrm{CH}_{2} \mathrm{CH}_{3}\right) . \mathrm{MS}\left(\mathrm{ESI}^{+}\right)(\mathrm{m} / \mathrm{z})=139\left\{[\mathrm{BMIM}]^{+}\right\} . \mathrm{IR}(\mathrm{KBr}) \mathrm{cm}^{-1}: 3404,3096,2954,1570,1463,1170$, $756,623$.

$[\mathrm{BMIM}]^{+} \mathrm{Cl}^{-}(76 \mathrm{~g}, 0.45 \mathrm{~mol})$ was dissolved in water $(30 \mathrm{~mL})$. Tetrafluoroboric acid $(63 \mathrm{~mL}, 0.5 \mathrm{~mol})$ was added dropwise to the above solution under stirring in an ice bath. The mixture was stirred at room temperature for $24 \mathrm{~h}$. The resulting $[\mathrm{BMIM}]^{+}\left[\mathrm{BF}_{4}\right]^{-}$was chilled in an ice bath and extracted with chilled dichloromethane (6 $\times 10 \mathrm{~mL}$ ). The dichloromethane fraction was washed with chilled water until the aqueous fraction $\mathrm{pH}$ became neutral and free of chloride $\left(\mathrm{AgNO}_{3}\right)$. The dichloromethane was removed by rotary evaporation and the crude product was mixed with charcoal and stirred for $24 \mathrm{~h}$ at room temperature. The charcoal was filtered off and the product was dried by heating under high vacuum. $[\mathrm{BMIM}]^{+}\left[\mathrm{BF}_{4}\right]^{-}$(3a) was obtained as a colorless viscous liquid (68g, 69\%). ${ }^{1} \mathrm{H}$ NMR (250 MHz, DMSO-d $\left.)_{6}\right) \delta: 9.07$ (s, 1H, NCHN), 7.75 (t, J=1.90 Hz, 1H, NCHCHN), $7.68(\mathrm{t}, J=1.90 \mathrm{~Hz}, 1 \mathrm{H}, \mathrm{NCHCHN}), 4.17$ (t, $\left.J=6.96 \mathrm{~Hz}, 2 \mathrm{H}, \mathrm{NCH}_{2}\right), 3.86\left(\mathrm{~s}, 3 \mathrm{H}, \mathrm{NCH}_{3}\right), 1.78$ (quint, $J=7.74$ $\mathrm{Hz}, 2 \mathrm{H}, \mathrm{NCH}_{2} \mathrm{CH}_{2}$ ), 1.27 (sext, $J=7.60 \mathrm{~Hz}, 2 \mathrm{H}, \mathrm{NCH}_{2} \mathrm{CH}_{2} \mathrm{CH}_{2}$ ), 0.91 (t, $J=7.74 \mathrm{~Hz}, 3 \mathrm{H}, \mathrm{NCH}_{2} \mathrm{CH}_{2} \mathrm{CH}_{2} \mathrm{CH}_{3}$ ). ${ }^{13} \mathrm{C}$ NMR (62.9 MHz, DMSO-d 6 ) $\delta: 136.34$ (NCHN), $123.52(\mathrm{NCHCHN}), 122.18$ (NCHCHN), 48.47 $\left(\mathrm{NCH}_{2} \mathrm{CH}_{2} \mathrm{CH}_{2} \mathrm{CH}_{3}\right), 35.62\left(\mathrm{NCH}_{3}\right), 31.29\left(\mathrm{NCH}_{2} \mathrm{CH}_{2} \mathrm{CH}_{2} \mathrm{CH}_{3}\right), 18.79\left(\mathrm{NCH}_{2} \mathrm{CH}_{2} \mathrm{CH}_{2} \mathrm{CH}_{3}\right), 13.16$ $\left(\mathrm{NCH}_{2} \mathrm{CH}_{2} \mathrm{CH}_{2} \mathrm{CH}_{3}\right) \cdot \mathrm{MS}\left(\mathrm{ESI}^{+}\right)(\mathrm{m} / \mathrm{z})=139\left\{[\mathrm{BMIM}]^{+}\right\} . \mathrm{IR}$ (neat) $\mathrm{cm}^{-1}: 3636,3159,2962,1573,1465,1079$, $1043,849,751,625$.

\subsubsection{1-Butyl-3-methylimidazolium hexafluorophosphate $\left\{[\mathrm{BMIM}]^{+}\left[\mathrm{PF}_{6}\right]^{-}\right\}$(3b) $[46]$}

This was prepared as described [46]. Yield: $21.45 \mathrm{~g}$ (75\%). ${ }^{1} \mathrm{H}$ NMR (250 MHz, DMSO-d 6 ) $\delta: 9.07$ (s, $1 \mathrm{H}$, $\mathrm{NCHN}), 7.73$ (t, $J=1.90 \mathrm{~Hz}, 1 \mathrm{H}, \mathrm{NCHCHN}), 7.66$ (t, $J=1.90 \mathrm{~Hz}, 1 \mathrm{H}, \mathrm{NCHCHN}), 4.17$ (t, $J=7.15 \mathrm{~Hz}, 2 \mathrm{H}$, 
$\mathrm{NCH}_{2}$ ), 3.86 (s, 3H, $\mathrm{NCH}_{3}$ ), 1.79 (quint, $J=7.71 \mathrm{~Hz}, 2 \mathrm{H}, \mathrm{NCH}_{2} \mathrm{CH}_{2}$ ), 1.28 (sext, $J=7.40 \mathrm{~Hz}, 2 \mathrm{H}$, $\mathrm{NCH}_{2} \mathrm{CH}_{2} \mathrm{CH}_{2}$ ), 0.91 (t, $J=7.22 \mathrm{~Hz}, 3 \mathrm{H}, \mathrm{NCH}_{2} \mathrm{CH}_{2} \mathrm{CH}_{2} \mathrm{CH}_{3}$ ). ${ }^{13} \mathrm{C}$ NMR (62.9 MHz, DMSO-d 6 ) $\delta: 136.40$ ( $\mathrm{NCHN}), 123.48$ ( $\mathrm{NCHCHN}), 122.12(\mathrm{NCHCHN}), 48.49\left(\mathrm{NCH}_{2} \mathrm{CH}_{2} \mathrm{CH}_{2} \mathrm{CH}_{3}\right), 35.57\left(\mathrm{NCH}_{3}\right), 31.26$ $\left(\mathrm{NCH}_{2} \mathrm{CH}_{2} \mathrm{CH}_{2} \mathrm{CH}_{3}\right), 18.69\left(\mathrm{NCH}_{2} \mathrm{CH}_{2} \mathrm{CH}_{2} \mathrm{CH}_{3}\right), 13.09\left(\mathrm{NCH}_{2} \mathrm{CH}_{2} \mathrm{CH}_{2} \mathrm{CH}_{3}\right) . \mathrm{MS}\left(\mathrm{ESI}^{+}\right)(\mathrm{m} / \mathrm{z})=$ $139\left\{[\mathrm{BMIM}]^{+}\right\}$. IR (neat) $\mathrm{cm}^{-1}: 3673,3173,2964,1573,1464,1168,882,832,745,627$.

\subsubsection{1-Butyl-3-methylimidazolium trifluoroacetate $\left\{[\mathrm{BMIM}]^{+}\left[\mathrm{CF}_{3} \mathrm{COO}^{-}\right\}\right.$(3c) $[42]$}

A solution of 2a (20.3 g, $0.12 \mathrm{~mol})$ in acetonitrile was mixed with a solution of sodium trifluoroacetate $(15.82 \mathrm{~g}$, $0.12 \mathrm{~mol})$ in acetonitrile/acetone $(1: 1)$. The mixture was stirred for $4 \mathrm{~h}$. The precipitate $(\mathrm{NaCl})$ was filtered off through celite and the solvent was removed. Dichloromethane $(20 \mathrm{~mL})$ was added into the product and the solution was filtered off again. Dichloromethane was removed by rotary evaporation and the product was kept in high vacuum at $60{ }^{\circ} \mathrm{C}$ for $3 \mathrm{~h}$. Yield: $23.18 \mathrm{~g} \mathrm{(79 \% ).}{ }^{1} \mathrm{H}$ NMR (250 MHz, DMSO-d 6 ) $\delta: 9.35$ (s, 1H, NCHN), 7.85 (t, $J=1.90 \mathrm{~Hz}, 1 \mathrm{H}, \mathrm{NCHCHN}), 7.77(\mathrm{t}, 1 \mathrm{H}, J=1.90 \mathrm{~Hz}, \mathrm{NCHCHN}), 4.18\left(\mathrm{t}, J=7.58 \mathrm{~Hz}, 2 \mathrm{H}, \mathrm{NCH}_{2}\right.$ ), 3.87 (s, $3 \mathrm{H}, \mathrm{NCH}_{3}$ ), 1.76 (quint, $J=7.58 \mathrm{~Hz}, 2 \mathrm{H}, \mathrm{NCH}_{2} \mathrm{CH}_{2}$ ), 1.24 (sext, $J=7.58 \mathrm{~Hz}, 2 \mathrm{H}, \mathrm{NCH}_{2} \mathrm{CH}_{2} \mathrm{CH}_{2}$ ), 0.88 (t, $\left.J=7.58 \mathrm{~Hz}, 3 \mathrm{H}, \mathrm{CH}_{3}\right) .{ }^{13} \mathrm{C}$ NMR (62.9 MHz, DMSO-d 6 ) $\delta: 136.80(\mathrm{NCHN}), 123.59(\mathrm{NCHCHN}), 122.29$ ( $\mathrm{NCHCHN}), 48.42\left(\mathrm{NCH}_{2} \mathrm{CH}_{2} \mathrm{CH}_{2} \mathrm{CH}_{3}\right), 35.58\left(\mathrm{NCH}_{3}\right), 31.38\left(\mathrm{NCH}_{2} \mathrm{CH}_{2} \mathrm{CH}_{2} \mathrm{CH}_{3}\right), 18.72\left(\mathrm{NCH}_{2} \mathrm{CH}_{2} \mathrm{CH}_{2} \mathrm{CH}_{3}\right)$, $13.13\left(\mathrm{NCH}_{2} \mathrm{CH}_{2} \mathrm{CH}_{2} \mathrm{CH}_{3}\right) . \mathrm{MS}\left(\mathrm{ESI}^{+}\right)(\mathrm{m} / \mathrm{z})=139\left\{[\mathrm{BMIM}]^{+}\right\} . \mathrm{IR}\left(\mathrm{cm}^{-1}\right)=3434,3092,2961,1698,1571,1466$, 1409, 1202, 1163, 1119, 825, 795, 716, 624.

\subsubsection{1-Butyl-2,3-dimethylimidazolium trifluoroacetate $\left\{[\mathrm{BMMIM}]^{+}\left[\mathrm{CF}_{3} \mathrm{COO}^{-}\right\}\right.$(3d)}

1-Butyl-2,3-dimethylimidazolium chloride $\left[\mathrm{BMMIM}^{+} \mathrm{Cl}^{-}\right.$(2b): 1,2-Dimethylimidazole (1b, $9.6 \mathrm{~g}, 0.1 \mathrm{~mol}$ ) was mixed with 2-chlorobutane $(12 \mathrm{~mL}, 0.11 \mathrm{~mol})$. The mixture was stirred at $80^{\circ} \mathrm{C}$ for $24 \mathrm{~h}$. The crude product was purified by recrystallisation in acetonitrile/ether and dried under reduced pressure. Yield: $12.65 \mathrm{~g}(65 \%) .{ }^{1} \mathrm{H}$ NMR (250 MHz, DMSO-d $)$ ) $7.71(\mathrm{~d}, J=1.90 \mathrm{~Hz}, 1 \mathrm{H}, \mathrm{NCHCHN}), 7.69(\mathrm{~d}, J=1.90 \mathrm{~Hz}, 1 \mathrm{H}, \mathrm{NCHCHN}), 4.12$ (t, $J=7.58 \mathrm{~Hz}, 2 \mathrm{H}, \mathrm{NCH}_{2}$ ), 3.76 (s, $3 \mathrm{H}, \mathrm{NCH}_{3}$ ), 2.59 (s, 3H, $\mathrm{CH}_{3}$ ), 1.69 (quint, $J=7.58 \mathrm{~Hz}, 2 \mathrm{H}, \mathrm{NCH}_{2} \mathrm{CH}_{2}$ ), 1.28 (sext, $J=7.58 \mathrm{~Hz}, 2 \mathrm{H}, \mathrm{NCH}_{2} \mathrm{CH}_{2} \mathrm{CH}_{2}$ ), 0.91 (t, $J=7.58 \mathrm{~Hz}, 3 \mathrm{H}, \mathrm{NCH}_{2} \mathrm{CH}_{2} \mathrm{CH}_{2} \mathrm{CH}_{3}$ ). ${ }^{13} \mathrm{C}$ NMR (62.9 MHZ, DMSO-d $\left._{6}\right) \delta: 144.15(\mathrm{NCN}), 122.25(\mathrm{NCHCHN}), 120.83(\mathrm{NCHCHN}), 47.18\left(\mathrm{NCH}_{2} \mathrm{CH}_{2} \mathrm{CH}_{2} \mathrm{CH}_{3}\right), 34.61$ $\left(\mathrm{NCH}_{3}\right), 31.16\left(\mathrm{NCH}_{2} \mathrm{CH}_{2} \mathrm{CH}_{2} \mathrm{CH}_{3}\right), 18.81\left(\mathrm{NCH}_{2} \mathrm{CH}_{2} \mathrm{CH}_{2} \mathrm{CH}_{3}\right), 13.36\left(\mathrm{CCH}_{3}\right), 9.13\left(\mathrm{NCH}_{2} \mathrm{CH}_{2} \mathrm{CH}_{2} \mathrm{CH}_{3}\right)$. MS $\left(\mathrm{ESI}^{+}\right)(\mathrm{m} / \mathrm{z})=153\left\{[\mathrm{BMMIM}]^{+}\right\} . \mathrm{IR}(\mathrm{KBr}) \mathrm{cm}^{-1}: 3402,3068,2967,1591,1543,1472,1253,1138,752$.

1-Butyl-2,3-dimethylimidazolium trifluoroacetate (3d) was prepared by following the same procedure as described for the synthesis of 3c. Yield: $7.12 \mathrm{~g}(94 \%) .{ }^{1} \mathrm{H}$ NMR $\left(250 \mathrm{MHz}, \mathrm{DMSO}-\mathrm{d}_{6}\right)$ ) 7.68 (d, $J=1.9 \mathrm{~Hz}$, $1 \mathrm{H}, \mathrm{NCHCHN}), 7.66$ (d, $J=1.9 \mathrm{~Hz}, 1 \mathrm{H}, \mathrm{NCHCH}-\mathrm{N}), 4.11$ (t, $\left.J=6.95 \mathrm{~Hz}, 2 \mathrm{H}, \mathrm{NCH}_{2}\right), 3.75\left(\mathrm{~s}, 3 \mathrm{H}, \mathrm{NCH}_{3}\right), 2.58$ (s, $3 \mathrm{H}, \mathrm{CH}_{3}$ ), 1.68 (quint, $J=7.58 \mathrm{~Hz}, 2 \mathrm{H}, \mathrm{NCH}_{2} \mathrm{CH}_{2}$ ) 1.28 (sext, $J=7.58 \mathrm{~Hz}, 2 \mathrm{H}, \mathrm{NCH}_{2} \mathrm{CH}_{2} \mathrm{CH}_{2}$ ), 0.90 (t, $J=$ $6.95 \mathrm{~Hz}, 3 \mathrm{H}, \mathrm{CH}_{2} \mathrm{CH}_{2} \mathrm{CH}_{2} \mathrm{CH}_{3}$ ). ${ }^{13} \mathrm{C}$ NMR (62.9 MHz, DMSO-d 6 ) $\delta: 144.30$ (NCN), 122.29 (NCHCHN), 120.83 ( $\mathrm{NCHCHN}), 47.21\left(\mathrm{NCH}_{2} \mathrm{CH}_{2} \mathrm{CH}_{2} \mathrm{CH}_{3}\right), 34.58\left(\mathrm{NCH}_{3}\right), 31.16\left(\mathrm{NCH}_{2} \mathrm{CH}_{2} \mathrm{CH}_{2} \mathrm{CH}_{3}\right), 18.83\left(\mathrm{NCH}_{2} \mathrm{CH}_{2} \mathrm{CH}_{2} \mathrm{CH}_{3}\right)$, $13.35\left(\mathrm{CCH}_{3}\right), 9.05\left(\mathrm{NCH}_{2} \mathrm{CH}_{2} \mathrm{CH}_{2} \mathrm{CH}_{3}\right)$. HRMS calcd for $\mathrm{C}_{9} \mathrm{H}_{17} \mathrm{~N}_{2}\left\{[\mathrm{BMMIM}]^{+}\right\} 153.1392$, found 153.1391. IR $(\mathrm{KBr}) \mathrm{cm}^{-1}: 3443,2967,1697,1540,1464,1423,1203,1172,1123,826,799,719$. 


\subsubsection{1-Methoxymethoxyethyl-3-methylimidazolium trifluoroacetate $\left\{[\mathrm{MeOMeOEtMIM}]^{+}\left[\mathrm{CF}_{3} \mathrm{COO}^{-}\right\}\right.$}

(3e)

1-Methoxymethoxyethyl-3-methylimidazolium bromide \{[MeOMeOEtMIM $\left.]^{+} \mathrm{Br}^{-}\right\}$(2c): 1-Methylimidazole (1a, $2.4 \mathrm{~g}, 0.03 \mathrm{~mol}$ ) was treated with 1-methoxymethoxyethyl bromide (5 g, $0.03 \mathrm{~mol})$. The mixture was stirred at $50{ }^{\circ} \mathrm{C}$ for $20 \mathrm{~h}$. The resulting product was washed with ethyl acetate $(2 \times 5 \mathrm{~mL})$ and dried in a high vacuum for 2 h at $80{ }^{\circ} \mathrm{C}$ to afford 2c. Yield: $7.2 \mathrm{~g}(97 \%) .{ }^{1} \mathrm{H}$ NMR $\left(250 \mathrm{MHz}, \mathrm{DMSO}_{6}\right) \delta: 9.31$ (s, 1H, NCHN), 7.88 (t, $J=$ $1.90 \mathrm{~Hz}, 1 \mathrm{H}, \mathrm{NCHCHN}), 7.85$ (t, $J=1.90 \mathrm{~Hz}, 1 \mathrm{H}, \mathrm{NCHCHN}), 4.58\left(\mathrm{~s}, 2 \mathrm{H}, \mathrm{OCH}_{2} \mathrm{OCH}_{3}\right), 4.42$ (t, $J=5.05 \mathrm{~Hz}$, $2 \mathrm{H}, \mathrm{NCH}_{2} \mathrm{CH}_{2} \mathrm{O}$ ), $3.89\left(\mathrm{~s}, 3 \mathrm{H}, \mathrm{NCH}_{3}\right), 3.82\left(\mathrm{t}, J=5.05 \mathrm{~Hz}, 2 \mathrm{H}, \mathrm{NCH}_{2} \mathrm{CH}_{2} \mathrm{O}\right), 3.16\left(\mathrm{~s}, 3 \mathrm{H}, \mathrm{OCH}_{3}\right) .{ }^{13} \mathrm{C} \mathrm{NMR}$ (62.9 MHz, DMSO-d $\left.{ }_{6}\right) \delta: 136.93(\mathrm{NCHN}), 123.49(\mathrm{NCHCHN}), 122.66(\mathrm{NCHCHN}), 95.25\left(\mathrm{OCH}_{2} \mathrm{O}\right), 69.61$ $\left(\mathrm{NCH}_{2} \mathrm{CH}_{2} \mathrm{O}\right), 57.95\left(\mathrm{OCH}_{3}\right), 48.59\left(\mathrm{NCH}_{2} \mathrm{CH}_{2} \mathrm{O}\right), 35.62\left(\mathrm{NCH}_{3}\right) \cdot \mathrm{MS}\left(\mathrm{ESI}^{+}\right)(\mathrm{m} / \mathrm{z})=171\left\{[\mathrm{MeOMeOEtMIM}]^{+}\right\}$. IR (neat) $\mathrm{cm}^{-1}: 3433,2961,1562,1466,1159,1115,1040,909,760$. For the synthesis of 3e, a similar method was followed as described for the synthesis of 3c. [MeOMeOEtMIM] ${ }^{+} \mathrm{Br}^{-}(7 \mathrm{~g}, 0.03 \mathrm{~mol})$ was reacted with $\mathrm{CF}_{3} \mathrm{COONa}(4.80 \mathrm{~g}, 0.03 \mathrm{~mol})$ to afford $3 \mathrm{e}(7.23 \mathrm{~g}, 81 \%) .{ }^{1} \mathrm{H}$ NMR $(250 \mathrm{MHz}$, DMSO-d 6 ) $\delta: 9.25(\mathrm{~s}, 1 \mathrm{H}$, $\mathrm{NCHN}), 7.82(\mathrm{t}, J=1.90 \mathrm{~Hz}, 1 \mathrm{H}, \mathrm{NCHCHN}), 7.76(\mathrm{t}, J=1.90 \mathrm{~Hz}, 1 \mathrm{H}, \mathrm{NCHCHN}), 4.55\left(\mathrm{~s}, 2 \mathrm{H}, \mathrm{OCH}_{2} \mathrm{OCH}_{3}\right)$, $4.40\left(\mathrm{t}, J=5.05 \mathrm{~Hz}, 2 \mathrm{H}, \mathrm{NCH}_{2} \mathrm{CH}_{2} \mathrm{O}\right), 3.88\left(\mathrm{~s}, 3 \mathrm{H}, \mathrm{NCH}_{3}\right), 3.81\left(\mathrm{t}, J=5.05 \mathrm{~Hz}, 2 \mathrm{H}, \mathrm{NCH}_{2} \mathrm{CH} \mathrm{H}_{2} \mathrm{O}\right), 3.16(\mathrm{~s}, 3 \mathrm{H}$,

$\left.\mathrm{OCH}_{3}\right){ }^{13} \mathrm{C}$ NMR (62.9 MHz, DMSO-d $\left.\mathrm{d}_{6}\right) \delta: 137.09(\mathrm{NCHN}), 123.47$ ( $\left.\mathrm{NCHCHN}\right), 122.55(\mathrm{NCHCHN}), 95.50$ $\left(\mathrm{OCH}_{2} \mathrm{O}\right), 64.93\left(\mathrm{NCH}_{2} \mathrm{CH}_{2} \mathrm{O}\right), 54.72\left(\mathrm{OCH}_{3}\right), 48.87\left(\mathrm{NCH}_{2} \mathrm{CH}_{2} \mathrm{O}\right), 35.68\left(\mathrm{NCH}_{3}\right)$. HRMS calcd for $\mathrm{C}_{8} \mathrm{H}_{15} \mathrm{~N}_{2} \mathrm{O}_{2}$ $\left\{[\mathrm{MeOMeOEtMIM}]^{+}\right\} 171.1134$, found 171.1138. IR (neat) $\mathrm{cm}^{-1}: 3429,3087,2953,1694,1429,1173,1047$, 831.

\subsubsection{1-Methoxyethyl-3-methylimidazolium trifluoroacetate $\left\{[\mathrm{MeOEtMIM}]^{+}\left[\mathrm{CF}_{3} \mathrm{COO}\right]\right\}$ (3f) [47]} 1-Methoxyethyl-3-methylimidazolium chloride $\left\{[\mathrm{MeOEtMIM}]^{+} \mathrm{Cl}^{-}\right\}$(2d) [41]: 1-methylimidazole (1a, $8 \mathrm{~mL}, 0.1$ mol) was added dropwise to 2-chloroethyl methyl ether $(25 \mathrm{~mL}, 0.27 \mathrm{~mol})$. The mixture was stirred and refluxed at $80{ }^{\circ} \mathrm{C}$ for $50 \mathrm{~h}$. The upper layer, 2-chloroethyl methyl ether, was decanted. The resulting ionic liquid was washed with 2-chloroethyl methyl ether $(2 \times 5 \mathrm{~mL})$ and evaporated. The crude product was dissolved in acetonitrile and recrystallized in acetonitrile/ether and dried under vacuum overnight. Yield: $17.59 \mathrm{~g} \mathrm{(99 \% ).}{ }^{1} \mathrm{H}$ NMR (250 MHZ, DMSO-d $\left.{ }_{6}\right) \delta: 9.57$ (s, 1H, NCHN), 7.91 (t, $\left.J=1.90 \mathrm{~Hz}, 1 \mathrm{H}, \mathrm{NCHCHN}\right), 7.85$ (t, $J=1.90 \mathrm{~Hz}$, $1 \mathrm{H}, \mathrm{NCHCHN}), 4.42\left(\mathrm{t}, J=5.05 \mathrm{~Hz}, 2 \mathrm{H}, \mathrm{NCH}_{2} \mathrm{CH}_{2} \mathrm{O}\right.$ ), 3.90 (s, $\left.3 \mathrm{H}, \mathrm{NCH}_{3}\right), 3.71$ (t, $J=5.05 \mathrm{~Hz}, 2 \mathrm{H}$, $\mathrm{NCH}_{2} \mathrm{CH}_{2} \mathrm{O}$ ), 3.24 (s, $\left.3 \mathrm{H}, \mathrm{OCH}_{3}\right) .{ }^{13} \mathrm{C}$ NMR (62.9 MHz, DMSO-d 6 ) $\delta: 136.93$ (NCHN), 123.33 (NCHCHN), $122.51(\mathrm{NCHCHN}), 69.52\left(\mathrm{NCH}_{2} \mathrm{CH}_{2} \mathrm{O}\right), 57.93\left(\mathrm{OCH}_{3}\right), 48.36\left(\mathrm{NCH}_{2} \mathrm{CH}_{2} \mathrm{O}\right), 35.61\left(\mathrm{NCH}_{3}\right) . \mathrm{MS}\left(\mathrm{ESI}^{+}\right)(\mathrm{m} / \mathrm{z})=$ $141\left\{[\mathrm{MeOEtMIM}]^{+}\right\}$. IR (KBr) cm ${ }^{-1}: 3391,3072,1570,1449,1177,1117,1013,832$.

For the synthesis of 3f, a similar method was followed as described for the synthesis of 3c. A solution of 2d $(36.06 \mathrm{~g}, 0.2 \mathrm{~mol})$ in acetonitrile was added to a solution of sodium trifluoroacetate $(27.76 \mathrm{~g}, 0.2 \mathrm{~mol})$ in acetone to afford $3 f(43.07 \mathrm{~g}, 83 \%) .{ }^{1} \mathrm{H}$ NMR (250 MHz, DMSO-d 6 ) $\delta: 9.22(\mathrm{~s}, 1 \mathrm{H}, \mathrm{NCHN}), 7.78$ (t, $J=1.90 \mathrm{~Hz}, 1 \mathrm{H}$, $\mathrm{NCHCHN}), 7.74(\mathrm{t}, J=1.90 \mathrm{~Hz}, 1 \mathrm{H}, \mathrm{NCHCH}-\mathrm{N}), 4.37$ (t, $\left.J=5.05 \mathrm{~Hz}, 2 \mathrm{H}, \mathrm{NCH}_{2} \mathrm{CH}_{2} \mathrm{O}\right), 3.88\left(\mathrm{~s}, 3 \mathrm{H}, \mathrm{NCH}_{3}\right)$, $3.69\left(\mathrm{t}, J=5.05 \mathrm{~Hz}, 2 \mathrm{H}, \mathrm{NCH}_{2} \mathrm{CH}_{2} \mathrm{O}\right), 3.26\left(\mathrm{~s}, 3 \mathrm{H}, \mathrm{OCH}_{3}\right) .{ }^{13} \mathrm{C} \mathrm{NMR}\left(62.9 \mathrm{MHz}, \mathrm{DMSO}-\mathrm{d}_{6}\right) \delta: 136.87(\mathrm{NCHN})$, $123.42(\mathrm{NCHCHN}), 122.58(\mathrm{NCHCHN}), 69.54\left(\mathrm{NCH}_{2} \mathrm{CH}_{2} \mathrm{O}\right), 57.96\left(\mathrm{OCH}_{3}\right), 48.54\left(\mathrm{NCH}_{2} \mathrm{CH}_{2} \mathrm{O}\right), 35.65$ $\left(\mathrm{NCH}_{3}\right) . \mathrm{MS}\left(\mathrm{ESI}^{+}\right)(\mathrm{m} / \mathrm{z})=141\left\{[\mathrm{MeOEtMIM}]^{+}\right\}$. IR (neat) $\mathrm{cm}^{-1}: 3436,3086,1695,1682,1573,1454,1191$, $1123,823,800$. 
4.2.7. 1-Methoxyethyl-3-methylimidazolium trifluoromethanesulfonate $[\mathrm{MeOEtMIM}]^{+}\left[\mathrm{CF}_{3} \mathrm{SO}_{3}\right]^{-}(3 \mathrm{~g})[42]$ A similar method was followed as described for the synthesis of 3c. [MeOEtMIM] ${ }^{+} \mathrm{Cl}^{-}$(2d, $\left.6.93 \mathrm{~g}, 0.04 \mathrm{~mol}\right)$ was reacted with $\mathrm{CF}_{3} \mathrm{SO}_{3} \mathrm{Na}(6.75 \mathrm{~g}, 0.04 \mathrm{~mol})$ to give the product $3 \mathbf{h}$. Yield: $8.92 \mathrm{~g}(79 \%)$. ${ }^{1} \mathrm{H}$ NMR $(250 \mathrm{MHz}$, DMSO-d $\left.\mathrm{d}_{6}\right) \delta: 9.10(\mathrm{~s}, 1 \mathrm{H}, \mathrm{NCHN}), 7.75(\mathrm{t}, J=1.90 \mathrm{~Hz}, 1 \mathrm{H}, \mathrm{NCHCHN}), 7.70$ (t, $\left.J=1.90 \mathrm{~Hz}, 1 \mathrm{H}, \mathrm{NCHCHN}\right)$, $4.35\left(\mathrm{t}, J=5.21 \mathrm{~Hz}, 2 \mathrm{H}, \mathrm{NCH}_{2}\right), 3.86\left(\mathrm{~s}, 3 \mathrm{H}, \mathrm{NCH}_{3}\right), 3.67\left(\mathrm{t}, J=5.05 \mathrm{~Hz}, 2 \mathrm{H}, \mathrm{CH}_{2} \mathrm{O}\right), 3.26\left(\mathrm{~s}, 3 \mathrm{H}, \mathrm{OCH}_{3}\right)$. ${ }^{13} \mathrm{C}$ NMR (62.9 MHz, DMSO-d $\left.\mathrm{d}_{6}\right) \delta: 136.76$ (NCHN), 123.41 (NCHCHN), 122.56 ( $\left.\mathrm{NCHCHN}\right), 69.52$ $\left(\mathrm{NCH}_{2} \mathrm{CH}_{2} \mathrm{O}\right), 57.98\left(\mathrm{OCH}_{3}\right), 48.59\left(\mathrm{NCH}_{2} \mathrm{CH}_{2} \mathrm{O}\right), 35.68\left(\mathrm{NCH}_{3}\right) . \mathrm{MS}\left(\mathrm{ESI}^{+}\right)(\mathrm{m} / \mathrm{z})=141\left\{[\mathrm{MeOEtMIM}]^{+}\right\}$. IR (neat) $\mathrm{cm}^{-1}: 3565,3112,2943,2899,1572,1452,1266,1163,1030,835$.

\subsubsection{1-Methoxyethyl-3-butylimidazolium trifluoromethanesulfonate $\left\{[\mathrm{MeOEtBuIM}]^{+}\left[\mathrm{CF}_{3} \mathrm{SO}_{3}\right]^{-}\right\}$(3h)} 1-methoxyethyl-3-butylimidazolium chloride (2e, $6.7 \mathrm{~g}, 0.03 \mathrm{~mol}$ ), prepared as described [42], was dissolved in acetone. $\mathrm{CF}_{3} \mathrm{SO}_{3} \mathrm{Na}(5.3 \mathrm{~g}, 0.03 \mathrm{~mol})$ dissolved in acetone was added to the above solution. Following that a similar procedure was followed as for the synthesis of 3c. Yield: $6.75 \mathrm{~g}(66 \%) .{ }^{1} \mathrm{H}$ NMR $\left(250 \mathrm{MHz}, \mathrm{DMSO}-\mathrm{d}_{6}\right)$

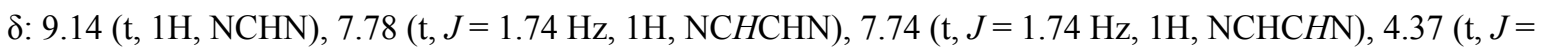
$4.74 \mathrm{~Hz}, 2 \mathrm{H}, \mathrm{NCH}_{2} \mathrm{CH}_{2} \mathrm{O}$ ), 4.20 (t, $J=7.27 \mathrm{~Hz}, 2 \mathrm{H}, \mathrm{NCH}_{2} \mathrm{CH}_{2} \mathrm{CH}_{2}$ ), 3.70 (t, $J=4.74 \mathrm{~Hz}, 2 \mathrm{H}, \mathrm{CH}_{2} \mathrm{O}$ ), 3.26 (s, $3 \mathrm{H}, \mathrm{OCH}_{3}$ ), 1.78 (quint, $J=7.27 \mathrm{~Hz}, 2 \mathrm{H}, \mathrm{NCH}_{2} \mathrm{CH}_{2} \mathrm{CH}_{2} \mathrm{CH}_{3}$ ), 1.25 (sext, $J=7.58 \mathrm{~Hz}, 2 \mathrm{H}, \mathrm{NCH}_{2} \mathrm{CH}_{2} \mathrm{CH}_{2} \mathrm{CH}_{3}$ ), $0.89\left(\mathrm{t}, J=7.58 \mathrm{~Hz}, 3 \mathrm{H}, \mathrm{NCH}_{2} \mathrm{CH}_{2} \mathrm{CH}_{2} \mathrm{CH}_{3}\right) .{ }^{13} \mathrm{C} \mathrm{NMR}\left(62.9 \mathrm{MHz}, \mathrm{DMSO}-\mathrm{d}_{6}\right) \delta: 136.18(\mathrm{NCHN}), 122.76$ ( $\mathrm{NCHCHN}), 122.22(\mathrm{NCHCHN}), 69.50\left(\mathrm{NCH}_{2} \mathrm{CH}_{2} \mathrm{O}\right), 57.94\left(\mathrm{OCH}_{3}\right), 48.74\left(\mathrm{NCH}_{2} \mathrm{CH}_{2} \mathrm{O}\right), 48.65$ $\left(\mathrm{NCH}_{2} \mathrm{CH}_{2} \mathrm{CH}_{2} \mathrm{CH}_{3}\right), 31.38\left(\mathrm{NCH}_{2} \mathrm{CH}_{2} \mathrm{CH}_{2} \mathrm{CH}_{3}\right), 18.74\left(\mathrm{NCH}_{2} \mathrm{CH}_{2} \mathrm{CH}_{2} \mathrm{CH}_{3}\right), 13.06\left(\mathrm{NCH}_{2} \mathrm{CH}_{2} \mathrm{CH}_{2} \mathrm{CH}_{3}\right) . \mathrm{MS}$ $\left(\mathrm{ESI}^{+}\right)(\mathrm{m} / \mathrm{z})=183\left\{[\mathrm{MeOEtBuIM}]^{+}\right\}$. HRMS calcd for $\mathrm{C}_{10} \mathrm{H}_{19} \mathrm{~N}_{2} \mathrm{O}\left\{[\mathrm{MeOEtBuIM}]^{+}\right\} 183.1498$, found 183.1493. IR (neat) $\mathrm{cm}^{-1}: 3559,3114,2971,1564,1461,1263,1159,1029,836$.

\subsection{Knoevenagel Condensation Reactions}

An aldehyde or a ketone $(5 \mathrm{mmol})$ and an alkylcyanoacetate $(5 \mathrm{mmol})$ were mixed in one of the ionic liquids (5 mmol) listed in Table 2. The reaction mixture was stirred for an appropriate time at $25{ }^{\circ} \mathrm{C}$ or $50{ }^{\circ} \mathrm{C}$ (Tables 2 and 3). When TLC analysis (diethyl ether/petroleum ether $=1 / 2,1 / 4$ or $1 / 6$ ) indicated the reaction was complete, the resulting product was extracted with ether $(3 \times 10 \mathrm{~mL})$, and the extraction was washed with water $(3 \times 10 \mathrm{~mL})$. Ether was removed by rotary evaporation. The product was further purified by recrystallization. To recycle the ionic liquid, the used ionic liquid was washed with ethyl acetate $(3 \times 10 \mathrm{~mL})$ and then was kept under high vacuum $(4 \mathrm{~mm} \mathrm{Hg})$ at $80^{\circ} \mathrm{C}$ for $3 \mathrm{~h}$.

\subsection{1. (E) Ethyl-2-cyano-3-phenyl-2-propenoate (9a) [31]}

Mp: 47-48 ${ }^{\circ} \mathrm{C} .{ }^{1} \mathrm{H}$ NMR $\left(250 \mathrm{MHz}\right.$, DMSO-d $\left.{ }_{6}\right) \delta: 8.39$ (s, $\left.1 \mathrm{H}, \mathrm{HC}=\mathrm{C}\right), 8.14-8.02(\mathrm{~m}, 2 \mathrm{H}, \mathrm{Ph}-\mathrm{H})$, 7.74-7.54 (m, $3 \mathrm{H}, \mathrm{Ph}-\mathrm{H}), 4.31$ (q, $\left.J=7.11 \mathrm{~Hz}, 2 \mathrm{H}, \mathrm{CH} 2 \mathrm{CH}_{3}\right), 1.30$ (t, $\left.J=7.11 \mathrm{~Hz}, 3 \mathrm{H}, \mathrm{CH}_{2} \mathrm{CH}_{3}\right) .{ }^{13} \mathrm{C}$ NMR $(62.9 \mathrm{MHz}$, DMSO-d $\left._{6}\right)$ 8: $161.72(\mathrm{COO}), 155.02(\mathrm{HC}=\mathrm{CCN}), 133.35\left(\mathrm{Ph}-\mathrm{C}_{4}\right), 131.27\left(\mathrm{Ph}-\mathrm{C}_{2+6}\right), 130.74\left(\mathrm{Ph}_{-} \mathrm{C}_{1}\right), 129.25$ $\left(\mathrm{Ph}-\mathrm{C}_{3+5}\right), 115.52(\mathrm{CN}), 102.52(\mathrm{HC}=C \mathrm{CN}), 62.33\left(\mathrm{OCH}_{2} \mathrm{CH}_{3}\right), 13.90\left(\mathrm{OCH}_{2} \mathrm{CH}_{3}\right)$. MS (ESI) $(\mathrm{m} / \mathrm{z}): 200(\mathrm{M}-\mathrm{H})$. IR (KBr) cm ${ }^{-1}: 3426,2980,2220,1724,1601,1438,1256,1196,1078,1005,769,678$.

\subsection{2. (E) Ethyl-2-cyano-3-(4-chlorophenyl)-2-propenoate (9b) [31]}

Mp: 91-92 ${ }^{\circ} \mathrm{C} .{ }^{1} \mathrm{H}$ NMR $\left(250 \mathrm{MHz}, \mathrm{DMSO}-\mathrm{d}_{6}\right) \delta: 8.39$ (s, 1H, H-C=C), $8.05(\mathrm{~d}, J=8.53 \mathrm{~Hz}, 2 \mathrm{H}, \mathrm{Ph}-\mathrm{H}), 7.66$ (d, 
$J=8.53 \mathrm{~Hz}, 2 \mathrm{H}, \mathrm{Ph}-\mathrm{H}), 4.31$ (q, $\left.J=7.11 \mathrm{~Hz}, 2 \mathrm{H}, \mathrm{CH}_{2} \mathrm{CH}_{3}\right), 1.30$ (t, $\left.J=7.11 \mathrm{~Hz}, 3 \mathrm{H}, \mathrm{CH}_{2} \mathrm{CH}_{3}\right) .{ }^{13} \mathrm{C}$ NMR $(62.9$ MHz, DMSO-d 6 ) $\delta: 161.56(\mathrm{COO}), 153.60(\mathrm{HC}=\mathrm{CCN}), 137.99\left(\mathrm{Ph}-\mathrm{C}_{4}\right), 132.39\left(\mathrm{Ph}-\mathrm{C}_{2+6}\right), 130.14\left(\mathrm{Ph}-\mathrm{C}_{1}\right)$, $129.41\left(\mathrm{Ph}-\mathrm{C}_{3+5}\right), 115.35(\mathrm{CN}), 103.11(\mathrm{HC}=\mathrm{CCN}), 62.42\left(\mathrm{OCH}_{2} \mathrm{CH}_{3}\right), 13.91\left(\mathrm{OCH}_{2} \mathrm{CH}_{3}\right) . \mathrm{MS}\left(\mathrm{ESI}^{-}\right)(\mathrm{m} / \mathrm{z}): 234$ (M-H). IR (KBr) cm ${ }^{-1}: 3434,2989,2225,1717,1609,1586,1488,1263,1200,1074,1007,827,755$.

\subsection{3. (E) Ethyl-2-cyano-3-(4-methylphenyl)-2-propenoate (9c) [53]}

Mp: 92-93 ${ }^{\circ} \mathrm{C} .{ }^{1} \mathrm{H}$ NMR (250 MHz, DMSO-d 6 ) $\delta: 8.32$ (s, $\left.1 \mathrm{H}, \mathrm{H}-\mathrm{C}=\mathrm{C}\right), 7.95(\mathrm{~d}, J=8.21 \mathrm{~Hz}, 2 \mathrm{H}, \mathrm{Ph}-\mathrm{H}), 7.39$ (d, $J=8.21 \mathrm{~Hz}, 2 \mathrm{H}, \mathrm{Ph}-\mathrm{H}), 4.30$ (q, $\left.J=7.11 \mathrm{~Hz}, 2 \mathrm{H}, \mathrm{CH}_{2} \mathrm{CH}_{3}\right), 2.39$ (s, $\left.3 \mathrm{H}, \mathrm{CH}_{3}\right), 1.30$ (t, $J=7.11 \mathrm{~Hz}, 3 \mathrm{H}$, $\left.\mathrm{CH}_{2} \mathrm{CH}_{3}\right) .{ }^{13} \mathrm{C}$ NMR (62.9 MHz, DMSO-d $\left.\mathrm{d}_{6}\right)$ \&: $161.95(\mathrm{COO}), 154.88(\mathrm{HC}=\mathrm{CCN}), 144.37\left(\mathrm{Ph}-\mathrm{C}_{4}\right), 130.94$ $\left(\mathrm{Ph}-\mathrm{C}_{2+6}\right), 129.90\left(\mathrm{Ph}-\mathrm{C}_{3+5}\right), 128.62\left(\mathrm{Ph}-\mathrm{C}_{1}\right), 115.75(\mathrm{CN}), 101.01(\mathrm{HC}=\mathrm{CCN}), 62.22\left(\mathrm{OCH}_{2} \mathrm{CH}_{3}\right), 21.30$ $\left(\mathrm{PhCH}_{3}\right), 13.93\left(\mathrm{OCH}_{2} \mathrm{CH}_{3}\right)$. MS (ESI $)(\mathrm{m} / \mathrm{z}): 214(\mathrm{M}-\mathrm{H}) . \mathrm{IR}(\mathrm{KBr}) \mathrm{cm}^{-1}: 3440,2997,2218,1727,1591,1273$, 1206, 1179, 1094, 816, 758, 494.

\subsection{4. (E) Ethyl-2-cyano-3-(4-nitrophenyl)-2-propenoate (9d) [31]}

Mp: $170-171{ }^{\circ} \mathrm{C} .{ }^{1} \mathrm{H}$ NMR $\left(250 \mathrm{MHz}\right.$, DMSO-d $\left.{ }_{6}\right): \delta=8.55$ (s, $\left.1 \mathrm{H}, \mathrm{H}-\mathrm{C}=\mathrm{C}\right), 8.40(\mathrm{~d}, J=9.00 \mathrm{~Hz}, 2 \mathrm{H}, \mathrm{Ph}-\mathrm{H})$, $8.24(\mathrm{~d}, J=9.00 \mathrm{~Hz}, 2 \mathrm{H}, \mathrm{Ph}-\mathrm{H}), 4.34\left(\mathrm{q}, J=7.11 \mathrm{~Hz}, 2 \mathrm{H}, \mathrm{CH}_{2} \mathrm{CH}_{3}\right), 1.32\left(\mathrm{t}, J=7.11 \mathrm{~Hz}, 3 \mathrm{H}, \mathrm{CH}_{2} \mathrm{CH}_{3}\right) .{ }^{13} \mathrm{C}$ NMR (62.9 MHz, $\left.\mathrm{CDCl}_{3}\right)$ \&: $161.10(\mathrm{COO}), 152.66(\mathrm{HC}=\mathrm{CCN}), 149.19\left(\mathrm{Ph}_{-} \mathrm{C}_{4}\right), 137.23\left(\mathrm{Ph}-\mathrm{C}_{1}\right), 131.64$ $\left(\mathrm{Ph}-\mathrm{C}_{2+6}\right), 124.13\left(\mathrm{Ph}-\mathrm{C}_{3+5}\right), 114.92(\mathrm{CN}), 106.62(\mathrm{HC}=\mathrm{CCN}), 62.71\left(\mathrm{OCH}_{2} \mathrm{CH}_{3}\right), 13.91\left(\mathrm{OCH}_{2} \mathrm{CH}_{3}\right)$. MS (ESI) (m/z): 245 (M-H). IR (KBr, cm $\left.{ }^{-1}\right): 3451,2991,2226,1718,1621,1590,1510,1342,1262,1205$, $1196,865,763,688$.

\subsection{5. (E) Ethyl-2-cyano-3-(4-carboxyphenyl)-2-propenoate (9e)}

Mp: $232-234{ }^{\circ} \mathrm{C} .{ }^{1} \mathrm{H}$ NMR $\left(250 \mathrm{MHz}\right.$, DMSO-d $\left.{ }_{6}\right) \delta: 13.42$ (s, $\left.1 \mathrm{H}, \mathrm{COOH}\right), 8.46(\mathrm{~s}, 1 \mathrm{H}, \mathrm{H}-\mathrm{C}=\mathrm{C}), 8.13(\mathrm{~d}, J=$ $8.67 \mathrm{~Hz}, 2 \mathrm{H}, \mathrm{Ph}-\mathrm{H}), 8.08$ (d, $J=8.53 \mathrm{~Hz}, 2 \mathrm{H}, \mathrm{Ph}-\mathrm{H}), 4.33$ (q, $\left.J=7.11 \mathrm{~Hz}, 2 \mathrm{H}, \mathrm{CH}_{2} \mathrm{CH}_{3}\right), 1.32$ (t, $J=7.11 \mathrm{~Hz}$, $\left.3 \mathrm{H}, \mathrm{CH}_{2} \mathrm{CH}_{3}\right) .{ }^{13} \mathrm{C}$ NMR (62.9 MHz, DMSO-d 6 ) $\delta: 166.36(\mathrm{COOH}), 161.40\left(\mathrm{COOC}_{2} \mathrm{H}_{5}\right), 153.80(\mathrm{HC}=\mathrm{CCN})$, $135.02\left(\mathrm{Ph}-\mathrm{C}_{1}\right), 134.22\left(\mathrm{Ph}_{-} \mathrm{C}_{4}\right), 130.69\left(\mathrm{Ph}_{-} \mathrm{C}_{2+6}\right), 129.82\left(\mathrm{Ph}-\mathrm{C}_{3+5}\right), 115.20(\mathrm{CN}), 104.73(\mathrm{NC}=C \mathrm{CN}), 62.52$ $\left(\mathrm{OCH}_{2} \mathrm{CH}_{3}\right), 13.90\left(\mathrm{OCH}_{2} \mathrm{CH}_{3}\right)$. HRMS calcd for $\mathrm{C}_{13} \mathrm{H}_{12} \mathrm{NO}_{4}[\mathrm{M}+\mathrm{H}] 246.0766$, found 246.0769. IR $(\mathrm{KBr}) \mathrm{cm}^{-1}$ : 3424, 2992, 2205, 1733, 1702, 1612, 1421, 1283, 1196, 1006, 850, 768, 686, 543.

\subsection{6. (E) Ethyl-2-cyano-3-(2-naphthyl)-2-propenoate (9f) [54]}

Mp: $111-112{ }^{\circ} \mathrm{C} .{ }^{1} \mathrm{H}$ NMR $\left(250 \mathrm{MHz}, \mathrm{DMSO}_{\mathrm{d}}\right) \delta: 8.60$ (s, 1H, H-C=C), 8.53(s, 1H, Ar-H), 8.26-7.99 (m, 4H, Ar-H), 7.78-7.61 (m, 2H, Ar-H), 4.34 (q, $\left.J=6.95 \mathrm{~Hz}, 2 \mathrm{H}, \mathrm{CH}_{2} \mathrm{CH}_{3}\right), 1.33$ (t, $\left.J=6.95 \mathrm{~Hz}, 3 \mathrm{H}, \mathrm{CH}_{2} \mathrm{CH}_{3}\right) .{ }^{13} \mathrm{C}$ NMR (62.9 MHz, DMSO-d ${ }_{6}$ ) 8: $161.90(\mathrm{COO}), 154.92(\mathrm{HC}=\mathrm{CCN}), 134.76$ (Ar-C), 134.43 (Ar-C), 132.29 (Ar-C), 129.27 (Ar-C), 129.24 (Ar-C), 128.95 (Ar-C), 128.91 (Ar-C), 127.81 (Ar-C), 127.41 (Ar-C), 124.48 (Ar-C), $115.77(\mathrm{CN}), 102.28(\mathrm{HC}=\mathrm{CCN}), 62.36\left(\mathrm{OCH}_{2} \mathrm{CH}_{3}\right), 13.97\left(\mathrm{OCH}_{2} \mathrm{CH}_{3}\right)$. MS (ESI) $(\mathrm{m} / \mathrm{z}): 250(\mathrm{M}-\mathrm{H})$. IR $(\mathrm{KBr}) \mathrm{cm}^{-1}: 3439,2981,2222,1724,1598,1248,1181,817,750,476$.

\subsection{7. (E) Methyl-2-cyano-3-(3-chlorophenyl)-2-propenoate (9g)}

Mp: 111-112 ${ }^{\circ} \mathrm{C} .{ }^{1} \mathrm{H}$ NMR $\left(250 \mathrm{MHz}, \mathrm{DMSO}_{6}\right.$ ) $\delta: 8.42$ (s, 1H, H-C=C), 8.09 (t, $\left.J=1.90 \mathrm{~Hz}, 1 \mathrm{H}, \mathrm{Ph}-\mathrm{H}\right)$, 8.05-7.99 (m, 1H, Ph-H), 7.73-7.58 (m, 2H, Ph-H), 3.87 (s, 3H, $\left.\mathrm{CH}_{3}\right) .{ }^{13} \mathrm{C}$ NMR (62.9 MHz, DMSO-d $\left.{ }_{6}\right) \delta$ : 
$161.89(\mathrm{COO}), 153.44(\mathrm{HC}=\mathrm{CCN}), 133.80\left(\mathrm{Ph}-\mathrm{C}_{3}\right), 133.24\left(\mathrm{Ph}-\mathrm{C}_{1}\right), 132.74\left(\mathrm{Ph}^{-\mathrm{C}_{5}}\right), 131.07\left(\mathrm{Ph}-\mathrm{C}_{2}\right), 130.16$ $\left(\mathrm{Ph}-\mathrm{C}_{4}\right), 128.94\left(\mathrm{Ph}-\mathrm{C}_{6}\right), 115.18(\mathrm{CN}), 103.95(\mathrm{HC}=\mathrm{CCN}), 53.42\left(\mathrm{OCH}_{3}\right)$. HRMS calcd for $\mathrm{C}_{11} \mathrm{H}_{8} \mathrm{NO}_{2} \mathrm{Cl}\left[\mathrm{M}^{+}\right]$ 221.0244, found 221.0247. IR (KBr) cm ${ }^{-1}: 3431,3059,2218,1722,1612,1562,1433,1272,1208,1093,781$, 680.

\subsection{8. (E) Methyl-2-cyano-3-(4-methylphenyl)-2-propenoate (9h)}

Mp: 108-110 ${ }^{\circ} \mathrm{C} .{ }^{1} \mathrm{H}$ NMR $\left(250 \mathrm{MHz}\right.$, DMSO-d $\left.{ }_{6}\right) \delta: 8.37$ (s, 1H, H-C=C), 7.99 (d, J=8.21 Hz, 2H, Ph-H), 7.41 $(\mathrm{d}, J=8.06 \mathrm{~Hz}, 2 \mathrm{H}, \mathrm{Ph}-\mathrm{H}), 3.86$ (s, $\left.3 \mathrm{H}, \mathrm{OCH}_{3}\right), 2.40$ (s, $\left.3 \mathrm{H}, \mathrm{CH}_{3}\right) .{ }^{13} \mathrm{C}$ NMR $\left(62.9 \mathrm{MHz}\right.$, DMSO-d $\left.{ }_{6}\right) \delta: 162.51$ (COO), $155.07(\mathrm{HC}=\mathrm{CCN}), 144.46\left(\mathrm{Ph}-\mathrm{C}_{4}\right), 130.08\left(\mathrm{Ph}-\mathrm{C}_{2+6}\right), 129.95\left(\mathrm{Ph}-\mathrm{C}_{3+5}\right), 128.64\left(\mathrm{Ph}-\mathrm{C}_{1}\right), 115.80(\mathrm{CN})$, $100.77(\mathrm{HC}=C \mathrm{CN}), 53.25\left(\mathrm{OCH}_{3}\right), 21.33\left(\mathrm{PhCH}_{3}\right)$. HRMS calcd for $\mathrm{C}_{12} \mathrm{H}_{11} \mathrm{NO}_{2}\left[\mathrm{M}^{+}\right] 201.0790$, found 201.0792. IR (KBr) cm $\mathrm{cm}^{-1}: 3434$, 2952, 2218, 1722, 1590, 1435, 1271, 1209, 1183, 1094, 811, 489.

\subsection{9. (E) Methyl-2-cyano-3-(4-methoxyphenyl)-2-propenoate (9i) [55]}

Mp: 102-103 ${ }^{\circ} \mathrm{C} .{ }^{1} \mathrm{H}$ NMR (250 MHz, DMSO-d 6 ) $\delta: 8.31$ (s, 1H, H-C=C), 8.09 (d, J=9.00 Hz, 2H, Ph-H), 7.15 (d, $J=9.00 \mathrm{~Hz}, 2 \mathrm{H}, \mathrm{Ph}-\mathrm{H}), 3.87$ (s, 3H, $\left.\mathrm{OCH}_{3}\right), 3.84$ (s, 3H, $\left.\mathrm{COOCH}_{3}\right) .{ }^{13} \mathrm{C}$ NMR $(62.9 \mathrm{MHz}$, DMSO-d 6 ) $\delta$ : $163.53(\mathrm{COO}), 162.84\left(\mathrm{Ph}_{-} \mathrm{C}_{4}\right), 154.51(\mathrm{HC}=\mathrm{CCN}), 133.51\left(\mathrm{Ph}-\mathrm{C}_{2+6}\right), 123.87\left(\mathrm{Ph}^{\left.-\mathrm{C}_{1}\right)}, 116.19(\mathrm{CN}), 114.91\right.$ $\left(\mathrm{Ph}-\mathrm{C}_{3+5}\right), 98.13(\mathrm{HC}=\mathrm{CCN}), 55.71\left(\mathrm{OCH}_{3}\right), 53.07\left(\mathrm{COOCH}_{3}\right)$. MS (ESI $)(\mathrm{m} / \mathrm{z}): 216(\mathrm{M}-\mathrm{H})$. IR $(\mathrm{KBr}) \mathrm{cm}^{-1}$ : $3434,2949,2213,1720,1585,1554,1509,1423,1257,1208,1168,1024,840,548$.

\subsubsection{0. (E) Methyl-2-cyano-3-(4-hydroxyphenyl)-2-propenoate (9j)}

Mp: $213-214{ }^{\circ} \mathrm{C} .{ }^{1} \mathrm{H}$ NMR (250 MHz, DMSO-d 6 ) $\delta: 10.89$ (s, $\left.1 \mathrm{H}, \mathrm{OH}\right), 8.26$ (s, $\left.1 \mathrm{H}, \mathrm{H}-\mathrm{C}=\mathrm{C}\right), 8.00$ (d, J= 8.85 $\mathrm{Hz}, 2 \mathrm{H}, \mathrm{Ph}), 6.95(\mathrm{~d}, J=8.64 \mathrm{~Hz}, 2 \mathrm{H}, \mathrm{Ph}), 3.83\left(\mathrm{~s}, 3 \mathrm{H}, \mathrm{CH}_{3}\right) .{ }^{13} \mathrm{C}$ NMR $(62.9 \mathrm{MHz}$, DMSO-d 6 ) $\delta: 163.10$ (COO), $162.08\left(\mathrm{Ph}_{-} \mathrm{C}_{1}\right), 154.79(\mathrm{HC}=\mathrm{CCN}), 133.00\left(\mathrm{Ph}_{-} \mathrm{C}_{2+6}\right), 122.42\left(\mathrm{Ph}_{-} \mathrm{C}_{4}\right), 116.45(\mathrm{CN}), 111.36\left(\mathrm{Ph}_{-} \mathrm{C}_{3+5}\right)$, $96.59(\mathrm{HC}=C \mathrm{CN}), 53.00\left(\mathrm{OCH}_{3}\right)$. HRMS calcd for $\mathrm{C}_{11} \mathrm{H}_{10} \mathrm{NO}_{3}[\mathrm{M}+\mathrm{H}] 204.0661$, found 204.0663. IR $(\mathrm{KBr})$ $\mathrm{cm}^{-1}: 3338,2222,1724,1590,1434,1270,1209,1170,1088,815,513$.

\subsubsection{Methyl-2-cyano-3-cyclopentanyl-2-propenoate (9k)}

Mp: 23-25 ${ }^{\circ} \mathrm{C} .{ }^{1} \mathrm{H}$ NMR $\left(250 \mathrm{MHz}, \mathrm{DMSO}-\mathrm{d}_{6}\right) \delta: 3.75\left(\mathrm{~s}, 3 \mathrm{H}, \mathrm{CH}_{3}\right), 2.91\left(\mathrm{t}, J=6.95 \mathrm{~Hz}, 2 \mathrm{H}, \mathrm{CH}_{2} \mathrm{C}=\mathrm{C}\right), 2.75$ (t, $\left.J=7.11 \mathrm{~Hz}, 2 \mathrm{H}, \mathrm{C}=\mathrm{CCH}_{2}\right), 1.85-1.69\left(\mathrm{~m}, 4 \mathrm{H}, \mathrm{CH}_{2} \mathrm{CH}_{2}\right) \cdot{ }^{13} \mathrm{C}$ NMR $\left(62.9 \mathrm{MHz}\right.$, DMSO-d $\left.\mathrm{d}_{6}\right): 188.79(\mathrm{C}=\mathrm{CCN})$, $161.77(\mathrm{COO}), 115.45(\mathrm{CN}), 99.15(\mathrm{C}=\mathrm{CCN}), 52.28\left(\mathrm{OCH}_{3}\right), 37.39\left(\mathrm{CH}_{2}\right), 35.25\left(\mathrm{CH}_{2}\right), 25.97\left(\mathrm{CH}_{2}\right), 24.56$ $\left(\mathrm{CH}_{2}\right)$. HRMS calcd for $\mathrm{C}_{9} \mathrm{H}_{11} \mathrm{NO}_{2}\left[\mathrm{M}^{+}\right] 165.0790$, found 165.0793. IR $(\mathrm{KBr}) \mathrm{cm}^{-1}: 3440,2957,2224,1727$, 1612, 1439, 1279, 1203, 1088, 1026, 773.

\subsubsection{Methyl-2-cyano-3-cyclohexanyl-2-propenoate (9l, liquid)}

${ }^{1} \mathrm{H}$ NMR (250 MHz, DMSO-d $\left.\mathrm{d}_{6}\right) \delta: 3.76\left(\mathrm{~s}, 3 \mathrm{H},-\mathrm{CH}_{3}\right), 2.95\left(\mathrm{t}, J=5.61 \mathrm{~Hz}, 2 \mathrm{H}, \mathrm{CH}_{2} \mathrm{C}=\mathrm{C}\right), 2.62(\mathrm{t}, J=5.85 \mathrm{~Hz}$, $\left.2 \mathrm{H}, \mathrm{C}=\mathrm{CCH}_{2}\right), 1.78-1.57\left(\mathrm{~m}, 6 \mathrm{H}, \mathrm{CH}_{2} \mathrm{CH}_{2} \mathrm{CH}_{2}\right) .{ }^{13} \mathrm{C}$ NMR $\left(62.9 \mathrm{MHz}, \mathrm{DMSO}-\mathrm{d}_{6}\right) \delta: 180.95(\mathrm{C}=\mathrm{CCN}), 161.85$ (COO), $115.41(\mathrm{CN}), 100.56(\mathrm{C}=\mathrm{CCN}), 52.53\left(\mathrm{OCH}_{3}\right), 36.19\left(\mathrm{CH}_{2}\right), 30.92\left(\mathrm{CH}_{2}\right), 28.12\left(\mathrm{CH}_{2}\right), 27.77\left(\mathrm{CH}_{2}\right)$, $24.83\left(\mathrm{CH}_{2}\right)$. HRMS calcd for $\mathrm{C}_{10} \mathrm{H}_{13} \mathrm{NO}_{2}\left[\mathrm{M}^{+}\right]$179.0946, found 179.0944. IR (neat) $\mathrm{cm}^{-1}: 3446,2940,2222$, 1734, 1601, 1437, 1269, 1216, 1096, 1012, 777. 


\subsubsection{3. (E) Methyl-2-cyano-3-phenyl-2-butyrate (9m, liquid)}

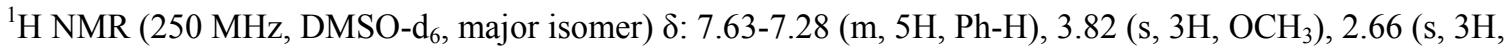
$\left.\mathrm{CH}_{3} \mathrm{C}=\mathrm{C}\right) .{ }^{13} \mathrm{C}$ NMR (62.9 MHz, DMSO-d $\mathrm{d}_{6}$, major isomer) $\delta: 173.02(\mathrm{COO}), 162.19(\mathrm{C}=\mathrm{CCN}), 138.49\left(\mathrm{Ph}-\mathrm{C}_{1}\right)$, $130.31\left(\mathrm{Ph}-\mathrm{C}_{4}\right), 128.55\left(\mathrm{Ph}-\mathrm{C}_{3+5}\right), 127.19\left(\mathrm{Ph}-\mathrm{C}_{2+6}\right), 116.15(\mathrm{CN}), 103.99(\mathrm{C}=\mathrm{CCN}), 52.77\left(\mathrm{OCH}_{3}\right), 26.65$ $\left(\mathrm{CH}_{3} \mathrm{C}=\mathrm{C}\right)$. HRMS calcd for $\mathrm{C}_{12} \mathrm{H}_{11} \mathrm{NO}_{2}\left[\mathrm{M}^{+}\right]$201.0790, found 201.0793. IR (neat) $\mathrm{cm}^{-1}: 3424,2976,2216$, $1719,1599,1437,1261,1194,1095,762,678$.

\subsubsection{4. (E) Ethyl-2-cyano-3-phenyl-2-butyrate (9n, liquid) [31]}

${ }^{1} \mathrm{H}$ NMR (250 MHz, DMSO-d 6 , major isomer) $\delta: 7.70-7.27$ (m, 5H, Ph-H), 4.29 (q, $J=6.95 \mathrm{~Hz}, 2 \mathrm{H}, \mathrm{CH}_{2} \mathrm{CH}_{3}$ ), $2.65\left(\mathrm{~s}, 3 \mathrm{H}, \mathrm{CH}_{3}\right), 1.29\left(\mathrm{t}, J=6.95 \mathrm{~Hz}, 3 \mathrm{H}, \mathrm{CH}_{2} \mathrm{CH}_{3}\right) .{ }^{13} \mathrm{C}$ NMR $(62.9 \mathrm{MHz}$, DMSO-d 6 , major isomer) $\delta: 172.67$ (COO), $161.71(C=\mathrm{CCN}), 140.13\left(\mathrm{Ph}_{-} \mathrm{C}_{1}\right), 133.12\left(\mathrm{Ph}-\mathrm{C}_{4}\right), 129.38\left(\mathrm{Ph}-\mathrm{C}_{3+5}\right), 126.52\left(\mathrm{Ph}_{-} \mathrm{C}_{2+6}\right), 116.10(\mathrm{CN})$, $104.29(\mathrm{C}=\mathrm{CCN}), 61.73\left(\mathrm{OCH}_{2} \mathrm{CH}_{3}\right), 26.48\left(\mathrm{CH}_{3} \mathrm{C}=\mathrm{C}\right), 13.87\left(\mathrm{OCH}_{2} \mathrm{CH}_{3}\right) . \mathrm{MS}\left(\mathrm{ESI}^{-}\right)(\mathrm{m} / \mathrm{z}): 214(\mathrm{M}-\mathrm{H}) . \mathrm{IR}$ (neat) $\mathrm{cm}^{-1}: 2985,2225,1729,1592,1442,1369,1242,1137,1046,764,700$.

\subsubsection{5. (E) Ethyl-2-cyano-2-hexenoate (9o, liquid) [56]}

${ }^{1} \mathrm{H}$ NMR (250 MHz, $\left.\mathrm{CDCl}_{3}\right) \delta: 7.65(\mathrm{t}, J=7.80 \mathrm{~Hz}, 1 \mathrm{H}, \mathrm{CH}=\mathrm{C}), 4.30$ (q, $J=7.20 \mathrm{~Hz}, 2 \mathrm{H}, \mathrm{OCH}_{2} \mathrm{CH}_{3}$ ), 2.51-2.60 (m, $\left.2 \mathrm{H}, \mathrm{CH}_{2} \mathrm{CH}_{2} \mathrm{CH}=\mathrm{C}\right), 1.57-1.68\left(\mathrm{~m}, 2 \mathrm{H}, \mathrm{CH}_{3} \mathrm{CH}_{2} \mathrm{CH}_{2} \mathrm{CH}=\mathrm{C}\right), 1.38$ (t, $\left.J=7.20 \mathrm{~Hz}, 3 \mathrm{H}, \mathrm{OCH}_{2} \mathrm{CH}_{3}\right)$, 0.99 (t, $\left.J=7.10 \mathrm{~Hz}, 3 \mathrm{H}, \mathrm{CH}_{3} \mathrm{CH}_{2} \mathrm{CH}_{2} \mathrm{CH}=\mathrm{C}\right) .{ }^{13} \mathrm{C} \mathrm{NMR}\left(62.9 \mathrm{MHz}, \mathrm{CDCl}_{3}\right) \delta: 163.46$ (COO), 161.10 $(\mathrm{HC}=\mathrm{CCN}), 113.66(\mathrm{CN}), 109.93(\mathrm{HC}=\mathrm{CCN}), 62.38\left(\mathrm{OCH}_{2} \mathrm{CH}_{3}\right), 33.75\left(\mathrm{CH}_{3} \mathrm{CH}_{2} \mathrm{CH}_{2} \mathrm{CH}=\mathrm{C}\right), 21.16$ $\left(\mathrm{CH}_{3} \mathrm{CH}_{2} \mathrm{CH}_{2} \mathrm{CH}=\mathrm{C}\right), 14.10\left(\mathrm{OCH}_{2} \mathrm{CH}_{3}\right), 13.72\left(\mathrm{CH}_{3} \mathrm{CH}_{2} \mathrm{CH}_{2} \mathrm{CH}=\mathrm{C}\right)$. MS (ESI $)(\mathrm{m} / \mathrm{z}): 166$ (M-H). IR (neat) $\mathrm{cm}^{-1}: 3436,2965,2231,1732,1270,760$.

\subsubsection{6. (E) Ethyl-2-cyano-3-(2-furyl)-2-propenoate (9p) [31]}

Mp: $92-93{ }^{\circ} \mathrm{C} .{ }^{1} \mathrm{H}$ NMR $\left(250 \mathrm{MHz}, \mathrm{CDCl}_{3}\right) \delta: 8.03(\mathrm{~s}, 1 \mathrm{H}, \mathrm{CH}=\mathrm{C}), 7.76(\mathrm{~d}, J=1.62 \mathrm{~Hz}, 1 \mathrm{H}$, furyl-H), $7.41(\mathrm{~d}, J$ $=4.0 \mathrm{~Hz}, 1 \mathrm{H}$, furyl-H), $6.69\left(\mathrm{dd}, J=4.0,1.62 \mathrm{~Hz}, 1 \mathrm{H}\right.$, furyl-H), $4.31\left(\mathrm{q}, J=7.10 \mathrm{~Hz}, 2 \mathrm{H}, \mathrm{OCH}_{2} \mathrm{CH}_{3}\right), 1.31(\mathrm{t}, J$ $\left.=7.10 \mathrm{~Hz}, 3 \mathrm{H}, \mathrm{OCH}_{2} \mathrm{CH}_{3}\right) .{ }^{13} \mathrm{C} \mathrm{NMR}\left(62.9 \mathrm{MHz}, \mathrm{CDCl}_{3}\right) \delta: 162.13(\mathrm{COO}), 149.97$ (furyl- $\mathrm{C}_{2}$ ), 148.13 (furyl- $\mathrm{C}_{5}$ ), $139.01\left(\mathrm{HC}=\mathrm{CCN}\right.$ ), 124.41 (furyl- $\left.\mathrm{C}_{3}\right), 115.22(\mathrm{CN}), 114.21$ (furyl- $\left.\mathrm{C}_{4}\right), 96.90(\mathrm{HC}=\mathrm{CCN}), 62.07\left(\mathrm{OCH}_{2} \mathrm{CH}_{3}\right)$, 13.91 $\left(\mathrm{OCH}_{2} \mathrm{CH}_{3}\right)$. MS (ESI) (m/z): $190(\mathrm{M}-\mathrm{H})$. IR (KBr) cm ${ }^{-1}: 3554,3410,2989,2406,1736,1621,1460,1218$, 779.

\section{Conflict of Interest}

The authors confirm that this article content has no conflict of interest.

\section{Acknowledgments}

Xiomei Hu thanks School of Life \& Health Sciences, Aston University for providing an International Scholarship. 


\section{References}

[1] Jones, G. Org. Reac., 1967, 15, 204-599.

[2] Sebti, S.; Smahi, A.; Solhy, A. Natural phosphate doped with potassium fluoride and modified with sodium nitrate: efficient catalysts for the Knoevenagel condensation. Tetrahedron Lett., 2002, 43, 1813-1815.

[3] Moison, H.; Texier-Boullet, F.; Foucaud, A. Knoevenagel, Witting and Witting-Horner reactions in the presence of magnesium-oxide or zinc-oxide. Tetrahedron, 1987, 43, 537.

[4] Brunel, D. Functionalized micelle-templated silicas (MTS) and their use as catalysts for fine chemicals. Micropor. Mesopor. Mater., 1999, 27, 329-344.

[5] Corma, A.; Fornes, V.; Martin-Aranda, R. M.; Reiy, F. Determination of bas properties of hydrotalcites - condensation of benzadehyde with ethyl acetoacetate. J. Catal., 1992, 134, 58.

[6] Corma, A.; Fornes, V.; Martin-Aranda, R. M.; Garcia, H.; Primo, J. Ceolites as base catalysts condensation of aldehydes with derivatives of malonic esters. Appl. Catal., 1990, 59, 237-248.

[7] Climent, M. J.; Corma, A.; Fornes, V.; Frau, A.; Guil-Lopez, R.; Iborra, S.; Primo, J. Alominophosphates oxynitrides as base catalysts: nature of the base sites and their catalytic implications. J. Catal., 1996, 163, 392-398.

[8] Aramendia, M. A.; Borau, V.; Jimenez, C.; Marinas, J. M.; Romero, F. J. Acidity of alkaline and alkaline-earth carbonates as heteogeneous catalysts for the Knoevenagel condensation between benzaldehyde and malnonitrile. Chem. Lett., 1995, 279-280.

[9] Reddy, B. M.; Patil, M. K.; Rao, K. N.; Reddy, G. K. An easy-to-use heterogenous promoted zirconia catalyst for Knoevenagel condensation in liquid phase under solvent-free conditions. J. Mol. Catal. A: Chem., 2006, 258, 302-307.

[10] Sowmiah, S.; Cheng, C. I.; Chu, Y.-H. Ionic liquids for green organic synthesis. Curr. Org. Chem., 2012, 74-95.

[11] Wasserscheid, P.; Welton, T.: Ionic Liquids in Synthesis Wiley: Weinheim, Germany, 2002.

[12] Suzuki, Y.; Wakatsuki, J.; Tsubaki, M.; Sato, M. Imidazolium-based chiral ionic liquids: synthesis and application. Tetrahedron, 2013, 69, 9690-9700.

[13] Teijeira, M.; Morales-Helguera, M. Synthesis of Oxacyclic derivatives using ionic liquids as a reaction medium. Curr. Org. Synth., 2012, 65-73.

[14] Jain, N.; Kumar, A.; Chauhan, S.; Chauhan, S. M. S. Chemical and biochemical transformation in ionic liquids. Tetrahedron 2005, 61, 1015-1060.

[15] Salunkhe, M. M.; Harjani, J. R.; Nara, S. J. Lewis acidic ionic liquids for the synthesis of electrophilic alkenes via the Knoevenagel condensation. Tetrahedron Lett., 2002, 43, 1127-1130.

[16] Liu, X. H.; Fan, J. C.; Liu, Y.; Shang, Z. C. L-Proline as an efficient and reusable promoter for the synthesis of coumarins in ionic liquid. Zhejiang University Science B, 2008, 9, 990.

[17] Wang, Y.; Shang, Z.; Wu, T.; Fan, J.; Ohsaka, T. Synthesis and theoretical study on proline-catalyzed Knoevenagel condensation in ionic liquid. J. Mol. Catal. A: Chem., 2006, $253,212-221$.

[18] Morrison, D. W.; Forbes, D. C.; Davis, J. H. Base-promoted reactions in ionic liquid solvents. The Knoevenagel and Robinson annulation reactions. Tetrahedron Lett., 2001, 42, 6053 6055. 
[19] Forbes, D. C.; Law, A. M.; Morrison, D. W. The Knoevenagel reaction: analysis and recycling of the ionic liquid medium. Tetrahedron Lett., 2006, 47, 1699-1703.

[20] Santamarta, F.; Verdia, P.; Tojo, E. A simple, efficient and green procedure for Knoevenagel reaction in [MMIm] $\left[\mathrm{MSO}_{4}\right]$ ionic liquid. Catal. Commun., 2008, 9, 1779-1781.

[21] Khan, F. A.; Dash, J.; Satapathy, R.; Upadhyay, K. S. Hydrotalcite catalysis in ioni liquid medium: a recyclable reaction system for heterogeneous Knoevenagel and nitroaldol condensation. Tetrahedron Lett., 2004, 45, 3055-3058.

[22] Lai, G.; Peng, J.; Li, J.; Qiu, H.; Jiang, J.; Jiang, K.; Shen, Y. Ionic liquids functionalised silica gel: novel catalyst and fixed solvent. Tetrahedron lett., 2006, 47, 6951-6953.

[23] Xin, X.; Guo, X.; Duan, F.; Lin, Y.; Sun, H. Efficient Knoevenagel condensation catalyzed by cyclic quanidium lactate ionic liquid as medium. Catal. Commun., 2007, 8, 115-117.

[24] Song, H.; Yu, Y.; Chen, X.; Li, X.; Xi, H. 2-Pyrrolidinecarboxylic acid ionic liquid catalyzed Knoevenagel condensation. Chinese J. Catal., 2012, 33, 666-669.

[25] Xu, D. Z.; Liu, Y.; Shi, S.; Y., W. A simple, efficient and green procedure for Knoevenagel condensation catalyzed by [C4dabco][Bf4] ionic liquid in water. Green Chem., 2010, 12, 514-517.

[26] Ying, A.; Ni, Y.; Xu, S.; Liu, S.; Yang, J.; Li, R. Noval DABCO based ionic liquids: Green and efficient catalysts with dual catalytic roles for aqueous Knoevenagel condensation. Ind. Eng. Chem. Res., 2014, 53, 5678-5682.

[27] Zhu, A.; Liu, R.; Li, L.; Li, L.; Wang, L.; Wang, J. Dual functions of $\mathrm{N}, \mathrm{N}$-dimethylethanolamnium-based ionic liquids for the Knoevenagel reactions at room temperature. Catal. Today, 2013, 200, 17-23.

[28] Valizadeh, H.; Gholipour, H. Imidazolium-based phosphinite ionic liquid (IL-OPPh2) as reusable catalyst and solvent for the knovevnagel condensation reaction. Synth. Commun., 2010, 40, 1477-1485.

[29] Hu, Y.; Chen, J.; Le, Z. G.; Zheng, Q. G. Organic reaction in ionic liquids: Ionic liquids ethylammonium nitrate promoted Knoevenagel condensation of aromatic aldehydes with active methylene compounds. Synth. Commun., 2005, 35, 739-744.

[30] Hu, Y.; Chen, Z. C.; Le, Z. G.; Zheng, Q. G. Organic reactions in ionic liquids: Ionic liquid promoted Knoevenagel condensation of aromatic aldehydes with (2-thio)barbituric acid. Synth. Commun., 2004, 34, 4521-4529.

[31] Su, C.; Chen, Z. C.; Zheng, Q. G. Organic reactions in ionic liquids: Knoevenagel condensation catalyzed by ethylenediammonium diacetate. Synthesis-Stuttgart, 2003, 555-559.

[32] Salunkhe, M. M.; Harjani, J. R.; Nara, S. J. Lewis acidic ionic liquids for the synthesis of electrophilic alkenes via the Knoevenagel condensation. Tetrahedron Lett., 2002, 43, 1127.

[33] Hu, Y.; Wei, P.; Huang, H.; Le, Z.-G.; Chen, Z.-C. Organic reactions in ionic liquids: Ionic liquid ethylammonium nitrate-promoted knoevenagel condensation of meldrum's acid with aromatic aldehydes. Synth. Commun., 2005, 35, 2955-2960.

[34] Yue, C.; Mao, A.; Wei, Y.; Lu, M. Knoevenagel condensation reaction catalyzed by task-specific ionic liquid under solvent-free conditions. Catal. Commun., 2008, 9, 1571-1574.

[35] Zhao, S.; Wang, X.; Zhang, L. Rapid and efficient Knoevenagel condensation catalyzed by a novel protic liquid under ultrasonic irradiation. RSC Adv., 2013, 3, 11691-11696. 
[36] Luo, Q.; Song, X.; Ji, M.; Park, S.; Hao, C.; Li, Y. Molecule size- and shape-selective Knoevenagel condensation over microporous Cu3(BTC)2 immobilized amino-functionalized basic ionic liquid catalyst. Appl. Catal. A: General, 2014, 478, 81-90.

[37] Ouyang, F.; Zhou, Y.; Li, Z.-M.; Hu, N.; Tao, D.-J. Tetrabutylphosphonium amino acid ionic liquids as efficient catalysts for solvent-free Knoevenagel condensation reactions. Korean $J$. Chem. Eng., 2014, 31, 1377-1383.

[38] Le, Z. G.; Chen, Z. C.; Hu, Y.; Zheng, Q. G. Organic reactions in ionic liquids: Ionic liquid-promoted efficient synthesis of N-alkyl and N-arylimides. Synthesis-Stuttgart, 2004, 995-998.

[39] Elaiwi, A.; Hitchcock, P. B.; Seddon, K. R.; Srinivasan, N.; Tan, Y.-M.; Welton, T.; Zora, J. A. Hydrogen bonding in imidazolium salts and its implications for ambient-temperature halogenoaluminate (III) ionic liquids. J. Chem. Soc. Dalton Trans., 1995, 3467-3472.

[40] Laali, K. K.; Gettwert, J. Electrophilic nitration of aromatics in ionic solvents. J. Org. Chem., 2001, 66, 35-40.

[41] Liu, Q.; Janssen, M. H. A.; Rantwijk, F.; Sheldon, R. A. Room-temperature ionic liquids that dissolve carbohydrates in high concentrations. Green Chem., 2005, 7, 39-42.

[42] Bonhote, P.; Dias, A.-P.; Papageorgiou, N.; Kalyanasundaram, K.; Gratzel, M. Hydrophobic, highlyconductive ambient-temperature molten salts. Inorg. Chem., 1996, 35, 1168-1178.

[43] Lucas, P.; Mehdi, N. E.; Belanger, D.; Breau, L. Expedient synthesis of symmetric aryl ketones and of ambient-temperature molten salts of imidazole. Synthsis-Stuttgart, 2000, 1253-1258.

[44] Chowdhury, S.; Mohan, R.; Scott, J. Reactivity of ionic liquids. Tetrahedron, 2007, 73, 2363-2389.

[45] Lancaster, N. L.; Welton, T.; Young, G. B. A study of halide nucleophilicity in ionic liquids. J. Chem. Soc., Perkin Trans. 2001, 2267-2270.

[46] Dupont, J.; Consorti, C. S.; Suarez, A. Z.; deSouza, R. F. Preparation of 1-butyl-3-methyl imidazolium-based room temperature ionic liquids. Org. Synth., 2002, 79, 236-239.

[47] Kumar, V.; Parmar, V. S.; Malhotra, S. V. Enhanced solubility and selective benzoylation of nucleosides in novel ionic liquid. Tetrahedron Lett., 2007, 48, 809-812.

[48] Chakraborti, A. K.; Roy, S. R.; Kumar, D.; Chopra, P. Catalytic application of room temperature ionic liquids: [bmin][MeSO4] as a recyclable catalyst for synthesis of bis(indolyl)methane. Ion-fishing by MALDI-TOF-TOF MS and MS/MS studies to probe the proposed mechanistic model of catalysis. Green Chem., 2008, 10, 1111-1118.

[49] Chakraborti, A. K.; Roy, S. R. Catalysis by Ionic Liquids. J. Am. Chem. Soc., 2009, 131, 6902-6903.

[50] Roy, S. R.; Chakraborti, A. K. Supermolecular assemblies in ionic liquid catalysis for Aza-Michael reaction. Org. Lett., 2010, 12, 3866-3869.

[51] Sarkar, A.; Roy, S. R.; Chakraborti, A. K. Ionic liquid catalysed reaction of thiols with alpha, beta-unsaturated carbonyl compounds - remarkable influence of the C-2hydrogen and the anion. Chem. Commun., 2011, 47, 4538-4540.

[52] Tietze, L. F.; Beifuss, U. Comprehensive Organic Synthesis. Trost, B. M. Ed.;Pergamon Press: Oxford, 1991; pp 341-392.

[53] Hu, Y.; Chen, J.; Le, Z. G.; Zheng, Q. G. Organic reactions in ionic liquids: Ionic liquids ethylammonium nitrate promoted Knoevenagel condensation of aromatic aldehydes with 
active methylene compounds. Synth. Commun., 2005, 35, 739-744.

[54] Leelavathi, P.; Kumar, S. R. Niobium (V) chloride catalyzed Knoevenagel condensation: An efficient protocol for the preparation of electrophilic alkenes. J. Mol. Catal. A: Chem., 2005, 240, 99-102.

[55] Brillon, D.; Sauve, G. Silica Gel-catalyzed Knoevenagel condensation of peptidyl cyanomethyl ketone with aromatic aldehydes and ketones. A novel michael acceptor functionality for C-modified peptides: the benzylidene and alkylidene cyanomethyl ketone function. J. Org. Chem., 1992, 57, 1838-1842.

[56] Ranu, B. C.; Jana, R. Ionic liquid as catalyst and reaction medium - A simple, efficient and green procedure for Knoevenagel condensation of aliphatic and aromatic carbonyl compounds using a task-specific basic ionic liquid. Eur. J. Org. Chem., 2006, 16, 3767-3770 


\section{Figures:}

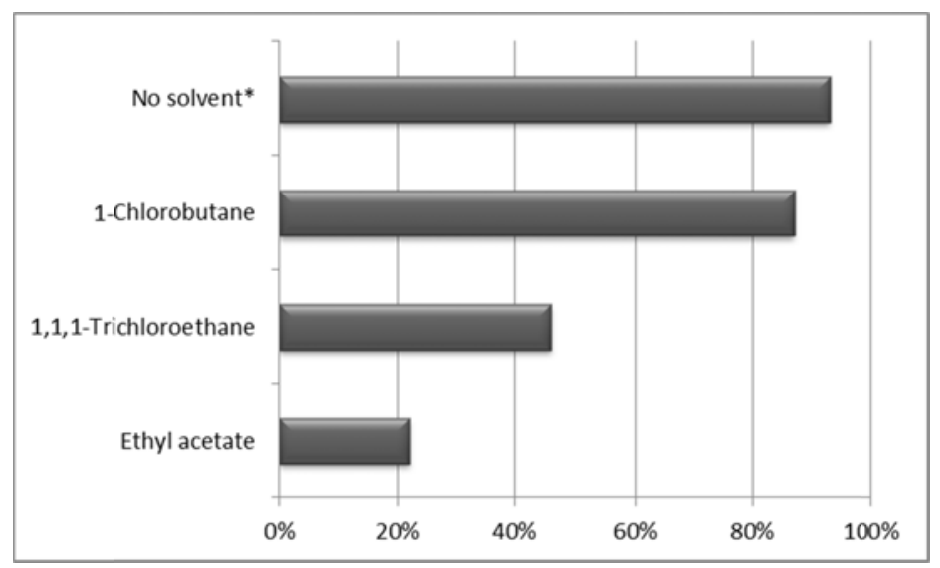

Figure 1. Comparison of various solvents for the preparation of $[\mathrm{BMIM}]^{+} \mathrm{Cl}^{-}(2 \mathbf{a})(*$ equal molar of the starting materials without addition of other solvents).

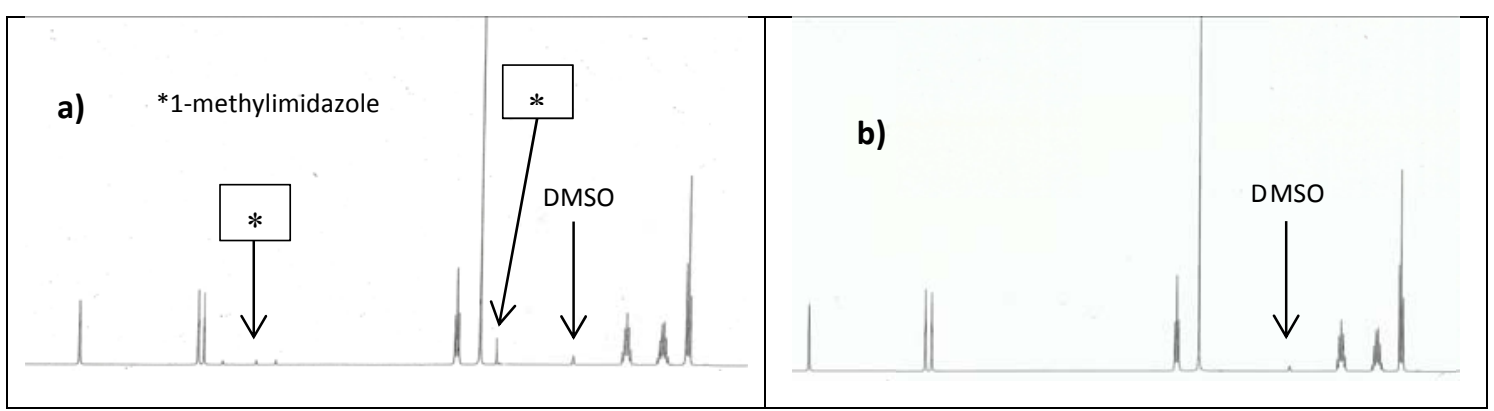

Figure 2. ${ }^{1} \mathrm{H}$ NMR spectrum of 1-butyl-3-methylimidazolium chloride, a) purified by heating under high vacuum; b) by recrystallization. 
Schemes:

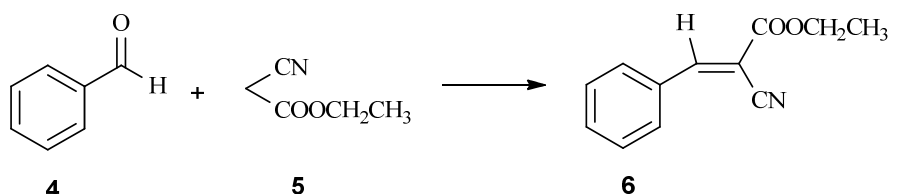

Scheme 1. Reaction of benzaldehyde with ethyl cyanoacetate in various ILs.

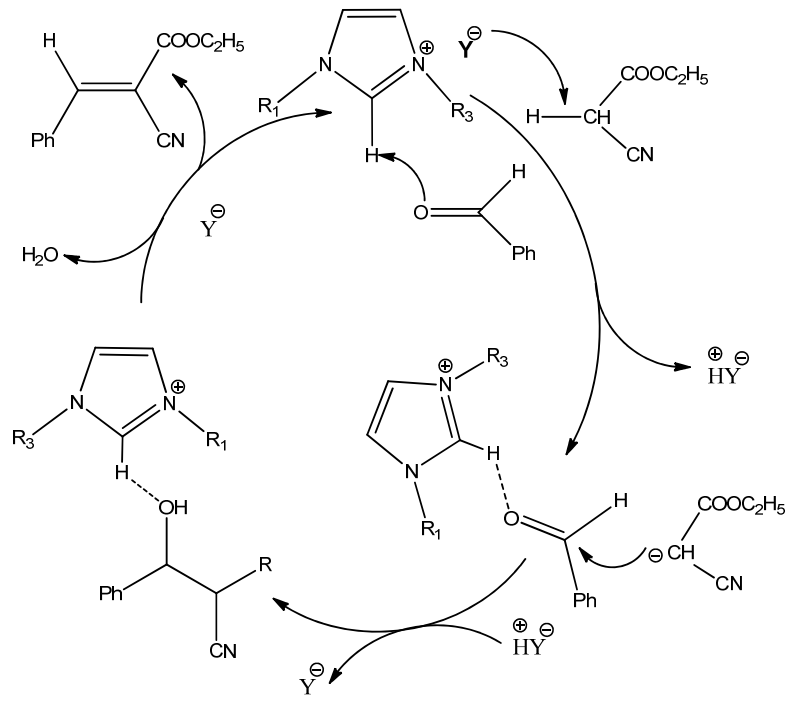

Scheme 2. Proposed mechanism for the Knoevenagel reaction. 


\section{Tables:}

Table 1. Synthesis of ionic liquids
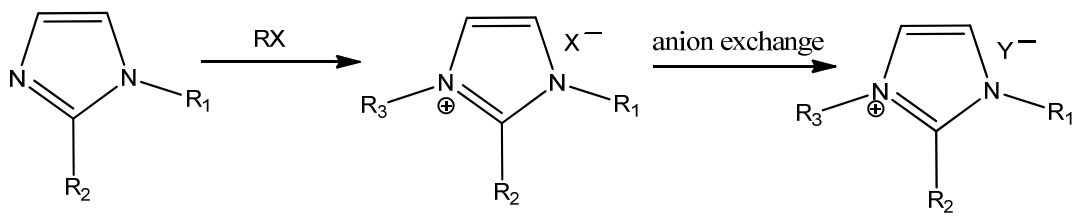

1a-c

2a-f

3a-i

\begin{tabular}{|c|c|c|c|c|c|}
\hline Compound No & $\mathbf{R}_{1}$ & $\mathbf{R}_{2}$ & $\mathbf{R}_{3}$ & $\mathbf{X}^{-}$ & $\mathbf{Y}^{-}$ \\
\hline $1 \mathrm{a}$ & $\mathrm{CH}_{3}$ & $\mathrm{H}$ & & & \\
\hline $1 \mathbf{b}$ & $\mathrm{CH}_{3}$ & $\mathrm{CH}_{3}$ & & & \\
\hline $1 \mathrm{c}$ & $\mathrm{n}-\mathrm{Bu}$ & $\mathrm{H}$ & & & \\
\hline 2a & $\mathrm{CH}_{3}$ & $\mathrm{H}$ & $\mathrm{n}-\mathrm{Bu}$ & $\mathrm{Cl}$ & \\
\hline $2 \mathbf{b}$ & $\mathrm{CH}_{3}$ & $\mathrm{CH}_{3}$ & $\mathrm{n}-\mathrm{Bu}$ & $\mathrm{Cl}$ & \\
\hline $2 \mathrm{c}$ & $\mathrm{CH}_{3}$ & $\mathrm{H}$ & $\mathrm{MeOMeOEt}$ & $\mathrm{Br}$ & \\
\hline $2 d$ & $\mathrm{CH}_{3}$ & $\mathrm{H}$ & $\mathrm{MeOEt}$ & $\mathrm{Cl}$ & \\
\hline $2 \mathbf{e}$ & $\mathrm{n}-\mathrm{Bu}$ & $\mathrm{H}$ & $\mathrm{MeOEt}$ & $\mathrm{Cl}$ & \\
\hline Зa & $\mathrm{CH}_{3}$ & $\mathrm{H}$ & $\mathrm{n}-\mathrm{Bu}$ & & $\mathrm{BF}_{4}$ \\
\hline $3 \mathbf{b}$ & $\mathrm{CH}_{3}$ & $\mathrm{H}$ & $\mathrm{n}-\mathrm{Bu}$ & & $\mathrm{PF}_{6}$ \\
\hline $3 c$ & $\mathrm{CH}_{3}$ & $\mathrm{H}$ & $\mathrm{n}-\mathrm{Bu}$ & & $\mathrm{CF}_{3} \mathrm{COO}$ \\
\hline $3 d$ & $\mathrm{CH}_{3}$ & $\mathrm{CH}_{3}$ & $\mathrm{n}-\mathrm{Bu}$ & & $\mathrm{CF}_{3} \mathrm{COO}$ \\
\hline $3 \mathbf{e}$ & $\mathrm{CH}_{3}$ & $\mathrm{H}$ & $\mathrm{MeOMeOEt}$ & & $\mathrm{CF}_{3} \mathrm{COO}$ \\
\hline $3 f$ & $\mathrm{CH}_{3}$ & $\mathrm{H}$ & $\mathrm{MeOEt}$ & & $\mathrm{CF}_{3} \mathrm{COO}$ \\
\hline $3 g$ & $\mathrm{CH}_{3}$ & $\mathrm{H}$ & $\mathrm{MeOEt}$ & & $\mathrm{CF}_{3} \mathrm{SO}_{3}$ \\
\hline $3 \mathbf{h}$ & $\mathrm{n}-\mathrm{Bu}$ & $\mathrm{H}$ & $\mathrm{MeOEt}$ & & $\mathrm{CF}_{3} \mathrm{SO}_{3}$ \\
\hline \multicolumn{6}{|c|}{ 3a: 1-Butyl-3-methylimidazolium tetrafluoroborate $\left\{[\mathrm{BMIM}]^{+}\left[\mathrm{BF}_{4}\right]^{-}\right\}$} \\
\hline \multicolumn{6}{|c|}{ 3b: 1-Butyl-3-methylimidazolium hexafluorophosphate $\left\{[\mathrm{BMIM}]^{+}\left[\mathrm{PF}_{6}\right]^{-}\right\}$} \\
\hline \multicolumn{6}{|c|}{ 3c: 1-Butyl-3-methylimidazolium trifluoroacetate $\left\{[\mathrm{BMIM}]^{+}\left[\mathrm{CF}_{3} \mathrm{COO}\right]^{-}\right\}$} \\
\hline \multicolumn{6}{|c|}{ 3d:1-Butyl-2,3-dimethylimidazolium trifluoroacetate $\left\{[\mathrm{BMMIM}]^{+} \mathrm{CF}^{2} \mathrm{COO}^{-}\right\}$} \\
\hline \multicolumn{6}{|c|}{ 3e:1-Methoxymethoxyethyl-3-methylimidazolium trifluoroacetate $\left\{[\mathrm{MeOMeOEtMIM}]^{+}\left[\mathrm{CF}_{3} \mathrm{COO}\right]^{-}\right\}$} \\
\hline \multicolumn{6}{|c|}{ 3f: 1-Methoxyethyl-3-methylimidazolium trifluoroacetate $\left\{[\mathrm{MeOEtMIM}]^{+}\left[\mathrm{CF}_{3} \mathrm{COO}^{-}\right\}\right.$} \\
\hline \multicolumn{6}{|c|}{ 3g: 1-Methoxyethyl-3-methylimidazolium trifluoromethanesulfonate $\left\{[\mathrm{MeOEtMIM}]^{+}\left[\mathrm{CF}_{3} \mathrm{SO}_{3}\right]^{-}\right\}$} \\
\hline \multicolumn{6}{|c|}{ 3h: 1-Methoxyethyl-3-butylimidazolium trifluoromethanesulfonate $\left\{[\mathrm{MeOEtBuIM}]^{+}\left[\mathrm{CF}_{3} \mathrm{SO}_{3}\right]^{-}\right\}$} \\
\hline
\end{tabular}


Table 2. Knoevenagel condensation of benzaldehyde with ethyl cyanoacetate in various ionic liquids ${ }^{\mathrm{a}}$

\begin{tabular}{|l|l|l|l|}
\hline Entry & Solvent & Reaction time & Yield (\%) $^{\mathbf{b}}$ \\
\hline $\mathbf{1}$ & {$[\mathrm{BMIM}]^{+}\left[\mathrm{BF}_{4}\right]^{-}(\mathbf{3 a})$} & $24 \mathrm{~h}$ & 85 \\
\hline $\mathbf{2}$ & {$[\mathrm{BMIM}]^{+}\left[\mathrm{PF}_{6}\right]^{-}(\mathbf{3 b})$} & $22 \mathrm{~h}$ & 63 \\
\hline $\mathbf{3}$ & {$[\mathrm{BMIM}]^{+}\left[\mathrm{CF}_{3} \mathrm{COO}\right]^{-}(\mathbf{3 c})$} & $1 \mathrm{~h}$ & 98 \\
\hline $\mathbf{4}$ & {$[\mathrm{BMMIM}]^{+}[\mathrm{CF} 3 \mathrm{COO}]^{-}(\mathbf{3 d})$} & $4 \mathrm{~h}$ & 62 \\
\hline $\mathbf{5}$ & {$[\mathrm{MeOMeOEtMIM}]^{+}\left[\mathrm{CF}_{3} \mathrm{COO}\right]^{-}(\mathbf{3 e})$} & $40 \mathrm{~min}$ & 95 \\
\hline $\mathbf{6}$ & {$\left[[\mathrm{MeOEtMIM}]^{+}\left[\mathrm{CF}_{3} \mathrm{COO}^{-}(\mathbf{3 f})\right.\right.$} & $40 \mathrm{~min}$ & 98 \\
\hline $\mathbf{7}$ & {$[\mathrm{MeOEtMIM}]^{+}\left[\mathrm{CF}_{3} \mathrm{SO}_{3}\right]^{-}(\mathbf{3 g})$} & $1 \mathrm{~h}$ & 81 \\
\hline $\mathbf{8}$ & {$[\mathrm{MeOEtBuIM}]^{+}\left[\mathrm{CF}_{3} \mathrm{SO}_{3}\right]^{-}(\mathbf{3 h})$} & $2 \mathrm{~h}$ & 54 \\
\hline${ }^{a}$ Reaction conditions: benzaldehyde $\left(5 \mathrm{mmol}^{2}\right.$, ethyl cyanoacetate $(5 \mathrm{mmol}), \mathrm{IL}(5 \mathrm{mmol})$ at $25^{\circ} \mathrm{C}$ & \\
${ }^{b}$ Isolated yield of products. &
\end{tabular}

Table 3. Knoevenagel condensation of aldehydes and ketones with methylene compounds in $[\mathrm{MeOEtMIM}]^{+}\left[\mathrm{CF}_{3} \mathrm{COO}^{-\mathrm{a}}\right.$

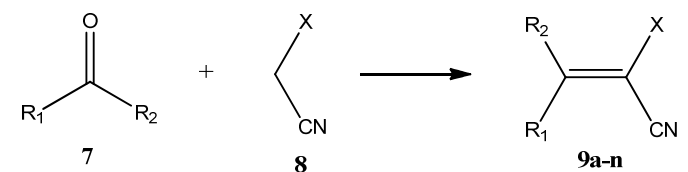

\begin{tabular}{|c|c|c|c|c|c|c|c|}
\hline Entry & R1 & R2 & $\mathbf{X}$ & Time & Temperature & Yield (\%) & Product \\
\hline 1 & $\mathrm{Ph}$ & $\mathrm{H}$ & COOEt & $40 \mathrm{~min}$ & $25^{\circ} \mathrm{C}$ & 98 & $9 a$ \\
\hline 2 & $4-\mathrm{Cl}-\mathrm{C}_{6} \mathrm{H}_{4}$ & $\mathrm{H}$ & COOEt & $5 \mathrm{~min}$ & $25^{\circ} \mathrm{C}$ & 97 & $9 \mathrm{~b}$ \\
\hline 3 & $4-\mathrm{Me}-\mathrm{C}_{6} \mathrm{H}_{4}$ & $\mathrm{H}$ & COOEt & $20 \mathrm{~min}$ & $25^{\circ} \mathrm{C}$ & 89 & $9 \mathrm{c}$ \\
\hline 4 & $4-\mathrm{NO}_{2}-\mathrm{C}_{6} \mathrm{H}_{4}$ & $\mathrm{H}$ & COOEt & $10 \mathrm{~min}$ & $25^{\circ} \mathrm{C}$ & 81 & $9 \mathrm{~d}$ \\
\hline 5 & $4-\mathrm{HOOC}-\mathrm{C}_{6} \mathrm{H}_{4}$ & $\mathrm{H}$ & COOEt & $50 \mathrm{~min}$ & $25^{\circ} \mathrm{C}$ & 77 & $9 \mathrm{e}$ \\
\hline 6 & 2-Naphthyl & $\mathrm{H}$ & COOEt & $15 \mathrm{~min}$ & $25^{\circ} \mathrm{C}$ & 98 & $9 f$ \\
\hline 7 & $3-\mathrm{Cl}-\mathrm{C}_{6} \mathrm{H}_{4}$ & $\mathrm{H}$ & COOMe & $2 \mathrm{~min}$ & $25^{\circ} \mathrm{C}$ & 99 & $9 g$ \\
\hline 8 & $4-\mathrm{Me}-\mathrm{C}_{6} \mathrm{H}_{4}$ & $\mathrm{H}$ & COOMe & $10 \mathrm{~min}$ & $25^{\circ} \mathrm{C}$ & 91 & $9 \mathrm{~h}$ \\
\hline 9 & $4-\mathrm{MeO}-\mathrm{C}_{6} \mathrm{H}_{4}$ & $\mathrm{H}$ & COOMe & $35 \mathrm{~min}$ & $25^{\circ} \mathrm{C}$ & 78 & $9 \mathrm{i}$ \\
\hline 10 & $4-\mathrm{HO}-\mathrm{C}_{6} \mathrm{H}_{4}$ & $\mathrm{H}$ & COOMe & $40 \mathrm{~min}$ & $25^{\circ} \mathrm{C}$ & 83 & $9 \mathrm{j}$ \\
\hline 11 & $-\left(\mathrm{CH}_{2}\right)_{4^{-}}$ & & COOMe & $48 \mathrm{~h}$ & $25^{\circ} \mathrm{C}$ & 85 & $9 \mathrm{k}$ \\
\hline 12 & $-\left(\mathrm{CH}_{2}\right)_{5^{-}}$ & & COOMe & $16 \mathrm{~h}$ & $25^{\circ} \mathrm{C}$ & 56 & 91 \\
\hline 13 & $\mathrm{Ph}$ & $\mathrm{CH}_{3}$ & COOMe & $48 \mathrm{~h}$ & $50^{\circ} \mathrm{C}$ & 55 & $9 \mathrm{~m}$ \\
\hline 14 & $\mathrm{Ph}$ & $\mathrm{CH}_{3}$ & COOEt & $48 \mathrm{~h}$ & $50^{\circ} \mathrm{C}$ & 47 & $9 n$ \\
\hline 15 & $\mathrm{n}-\mathrm{C}_{3} \mathrm{H}_{7}$ & $\mathrm{H}$ & COOEt & $2 \mathrm{~h}$ & $25^{\circ} \mathrm{C}$ & 82 & 90 \\
\hline 16 & 2-Furyl & $\mathrm{H}$ & COOEt & $80 \mathrm{~min}$ & $25^{\circ} \mathrm{C}$ & 85 & $9 p$ \\
\hline
\end{tabular}


Table 4. Knoevenagel condensation of benzaldehyde with ethyl cyanoacetate

\begin{tabular}{|l|l|}
\hline Ionic liquid & Yield (\%) \\
\hline$[\mathrm{MeOEtMIM}]^{+}\left[\mathrm{CF}_{3} \mathrm{COO}\right]^{-}$(Fresh) & 98 \\
\hline$[\mathrm{MeOEtMIM}]^{+}\left[\mathrm{CF}_{3} \mathrm{COO}\right]^{-}$(cycle1) & 94 \\
\hline$[\mathrm{MeOEtMIM}]^{+}\left[\mathrm{CF}_{3} \mathrm{COO}\right]^{-}$(cycle2) & 91 \\
\hline$[\mathrm{MeOEtMIM}]^{+}\left[\mathrm{CF}_{3} \mathrm{COO}\right]^{-}$(cycle3) & 88 \\
\hline$[\mathrm{MeOEtMIM}]^{+}\left[\mathrm{CF}_{3} \mathrm{COO}\right]^{-}$(cycle4) & 85 \\
\hline$[\mathrm{MeOEtMIM}]^{+}\left[\mathrm{CF}_{3} \mathrm{COO}\right]^{-}$(cycle5) & 86 \\
\hline
\end{tabular}

